\title{
Star formation and gas in the minor merger UGC 10214 ${ }^{\star}$
}

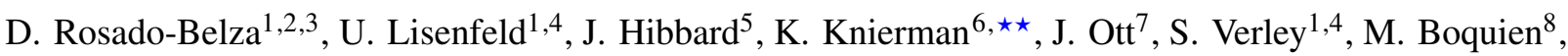 \\ T. Jarrett ${ }^{9}$, and C. K. $\mathrm{Xu}^{10,11}$
}

\author{
1 Departamento de Física Teórica y del Cosmos, Facultad de Ciencias, University of Granada, Av. Fuentenueva s/n, 18071 Granada \\ e-mail: darobel@correo.ugr.es \\ 2 Instituto de Astrofísica de Canarias, Vía Láctea s/n, 38205 La Laguna, Spain \\ 3 Departamento de Astrofísica, University of La Laguna, 38206 La Laguna, Spain \\ ${ }^{4}$ Instituto Carlos I, Facultad de Ciencias, University of Granada, Av. Fuentenueva s/n, 18071 Granada, Spain \\ 5 National Radio Astronomy Observatory, Charlottesville, VA 22903, USA \\ ${ }^{6}$ School of Earth \& Space Exploration, Arizona State University, 550 E. Tyler Mall, Room PSF-686, PO Box 876004, Tempe, AZ \\ 85287-6004, USA \\ 7 National Radio Astronomy Observatory, 1003 Lopezville Road, Socorro, NM 87801, USA \\ 8 Unidad de Astronomía, Fac. Cs. Básicas, Universidad de Antofagasta, Avda. U. de Antofagasta, 02800 Antofagasta, Chile \\ 9 Astrophysics, Cosmology and Gravity Centre, Astronomy Department, University of Cape Town, Private Bag X3, Rondebosch \\ 7701, South Africa \\ 10 National Astronomical Observatories, Chinese Academy of Sciences, Beijing 1000012, PR China \\ 11 South American centre for Astronomy, CAS, Camino El Observatorio 1515, Las Condes, Santiago, Chile
}

Received 18 July 2018 / Accepted 20 November 2018

\begin{abstract}
Minor mergers play a crucial role in galaxy evolution. UGC 10214 (the Tadpole galaxy) is a prime example of this process in which a dwarf galaxy has interacted with a large spiral galaxy $250 \mathrm{Myr}$ ago and produced a perturbed disc and a giant tidal tail. We used a multi-wavelength dataset that partly consists of new observations $(\mathrm{H} \alpha, \mathrm{HI}$, and $\mathrm{CO}$ ) and partly of archival data to study the present and past star formation rate (SFR) and its relation to the gas and stellar mass at a spatial resolution down to $4 \mathrm{kpc}$. UGC 10214 is a high-mass (stellar mass $\left.M_{\star}=1.28 \times 10^{11} M_{\odot}\right)$ galaxy with a low gas fraction $\left(M_{\mathrm{gas}} / M_{\star}=0.24\right)$, a high molecular gas fraction $\left(M_{\mathrm{H}_{2}} / M_{\mathrm{HI}}=0.4\right)$, and a modest SFR $\left(2-5 M_{\odot} \mathrm{yr}^{-1}\right)$. The global SFR compared to its stellar mass places UGC 10214 on the galaxy main sequence (MS). The comparison of the molecular gas mass and current SFR gives a molecular gas depletion time of about $\sim 2 \mathrm{Gyr}$ (based on $\mathrm{H} \alpha$ ), comparable to those of normal spiral galaxies. Both from a comparison of the $\mathrm{H} \alpha$ emission, tracing the current SFR, and far-ultraviolet (FUV) emission, tracing the recent SFR during the past tens of Myr, and also from spectral energy distribution fitting with CIGALE, we find that the SFR has increased by a factor of about 2-3 during the recent past. This increase is particularly noticeable in the centre of the galaxy where a pronounced peak of the $\mathrm{H} \alpha$ emission is visible. A pixel-to-pixel comparison of the SFR, molecular gas mass, and stellar mass shows that the central region has had a depressed FUV-traced SFR compared to the molecular gas and the stellar mass, whereas the $\mathrm{H} \alpha$-traced SFR shows a normal level. The atomic and molecular gas distribution is asymmetric, but the position-velocity diagram along the major axis shows a pattern of regular rotation. We conclude that the minor merger has most likely caused variations in the SFR in the past that resulted in a moderate increase of the SFR, but it has not perturbed the gas significantly so that the molecular depletion time remains normal.
\end{abstract}

Key words. galaxies: interactions - ISM: molecules - galaxies: evolution - galaxies: ISM - galaxies: star formation galaxies: individual: UGC 10214

\section{Introduction}

The question of how galaxies, in particular their star formation (SF) activity, evolve through cosmic time and how they build up their stellar mass, $M_{\star}$, has been the centre of numerous studies in the recent literature. Data from large galaxy surveys have established a relation between the star formation rate (SFR) and stellar mass of galaxies (e.g. Brinchmann et al. 2004), which holds out to high redshift, although with a different normalisation (e.g., Noeske et al. 2007). This relation shows a bimodality between active star-forming galaxies ("blue cloud") and quiescent galaxies ("red sequence") with a lower galaxy density in

\footnotetext{
* Data are only available at the CDS via anonymous ftp to cdsarc.u-strasbg.fr (130.79.128.5) or via http://cdsarc. u-strasbg.fr/viz-bin/qcat?J/A+A/623/A154

$\star \star$ NSF Astronomy and Astrophysics Postdoctoral Fellow.
}

the intermediate, possibly transitioning region ("green valley"). The relation between the SFR and $M_{\star}$ of the star-forming galaxies is also referred to as the main sequence (MS) of galaxies and can be taken as the "norm", whereas galaxies above and below have an excess or deficiency of SF for their stellar mass. It is still an open question how galaxies evolve along and across the MS and what processes are mainly responsible for their transition towards the red sequence. Various processes have been suggested, including gravitational interaction (see below), the presence of an active galactic nucleus (AGN; e.g. Ellison et al. 2016; Argudo-Fernández et al. 2016), and gas consumption or perturbation (e.g. Saintonge et al. 2016; Lisenfeld et al. 2017).

Gravitational interaction is an important process for the evolution of galaxies in clusters (Dressler 1980; Moore et al. 1996), groups (Hickson et al. 1992; Lisenfeld et al. 2017), triplets (Duplancic et al. 2015; Argudo-Fernández et al. 2015), and pairs 
(Ellison et al. 2010; Argudo-Fernández et al. 2015). Galaxies with nearby companions have shown alterations in their morphologies, star formation rates (SFRs), and gas reservoirs. In the most extreme cases, two or more galaxies may collide and their evolution may be severely altered through galaxy mergers. Major mergers between two galaxies of similar mass may result in the formation of a single massive elliptical galaxy (Toomre 1977; Schweizer 1982; Bell et al. 2006). Although less dramatic, minor mergers between galaxies of unequal mass (mass ratio $\lesssim$ 0.3 , Bournaud et al. 2005) might also greatly affect the evolution of both galaxies, in some cases leading to the formation of tidal tails and tidal dwarf galaxies (Bournaud \& Duc 2006; Kaviraj et al. 2012). The way in which two galaxies collide will determine the particular outcome of the remnant. To reach an insight into the consequences of a peculiar collision, the study of specific minor mergers, as an example of a simple collision scene, is necessary.

The Tadpole galaxy (UGC 10214, VV 29, or Arp 188) is a massive galaxy that has experienced a minor merger (where the remnant of the intruder galaxy represents less than $10 \%$ of the Tadpole gas mass, see Sect. 4) several hundreds of Myr ago. The encounter has distorted the Tadpole disc and caused gas to be ejected in a long $(\sim 110 \mathrm{kpc})$ tidal tail. Briggs et al. (2001) studied the atomic hydrogen (HI) gas distribution and kinematics of the system and identified three main dynamical components: VV 29a, which corresponds to the Tadpole disc; VV 29b, the tidal tail; and VV 29c, the dwarf galaxy. Tran et al. (2003) and de Grijs et al. (2003) analysed images taken with the Advanced Camera for Surveys installed on the Hubble Space Telescope (ACS/HST) and found a large number of young star cluster in the tail and disc. The ages of these clusters, which range from younger than $10 \mathrm{Myr}$ to $150-200 \mathrm{Myr}$, are younger than their estimate of the dynamical age of the interaction of $\sim 250 \mathrm{Myr}$, based on the length of the tail and expected ejection velocity of the gas (close to a rotation velocity of $400 \mathrm{~km} \mathrm{~s}^{-1}$ ). Jarrett et al. (2006) examined the star formation (SF) in UGC 10214 using optical and infrared imaging. They revealed several infraredbright hot spots in the spiral arms and little SF in the central region. They measured a global SFR of $2-4 M_{\odot} \mathrm{yr}^{-1}$.

These previous studies have shown that the Tadpole galaxy is an excellent object for investigating the effect of a minor interaction in the interstellar medium (ISM) and the SF in the host galaxy. However, so far, we lack data for the molecular gas as a crucial ingredient for SF, as well as multi-wavelength data allowing an analysis of the SF history in this object. In order to fill this gap, we carried out a multi-wavelength analysis of UGC 10214, including a large set of archival images from ultraviolet (UV) to submillimeter wavelengths, new HI data at a higher spatial resolution than previous data (Briggs et al. 2001), new CO data tracing the molecular gas, and an $\mathrm{H} \alpha$ image that shows the distribution of the current SF. The main goal of this paper is to better understand the present and past SF and its relation to the neutral (atomic and molecular) gas in this galaxy, which has suffered a minor interaction $\sim 250 \mathrm{Myr}$ ago in order to evaluate the impact of this event on the galaxy evolution.

This paper is organised as follows. In Sect. 2 we present the complete set of data we used in our study. Section 3 gives a description of the three methods we employed: aperture photometry of the galaxy disc as a whole and divided into three different regions; a spectral energy distribution (SED) modelling using the Code Investigating GALaxy Emission (CIGALE), and a pixel-by-pixel analysis of the three regions defined for the aperture photometry. Section 4 presents the results of the distribution and kinematics of the atomic and molecular gas and of the SFR and stellar mass. Additionally, we study the relation between these quantities on both a global and spatially resolved scale. In Sect. 5 we discuss the results of the previous section and reconstruct the star formation history of UGC 10214. We also discuss its future evolution. Finally, we present a brief summary and our conclusions in Sect. 6. We adopt a distance of $134.2 \pm 9.4 \mathrm{Mpc}$ (Mould et al. 2000) for this galaxy.

\section{Data}

An ample set of data is available to study UGC 10214, which covers a broad wavelength range from the UV to radio. Parts of the data (observations of $\mathrm{H} \alpha, \mathrm{CO}$, and $\mathrm{HI}$ ) are from our own observations and the rest are from public archives. In this section we describe the data and, when applicable, their reduction. In Table 1 we present a summary of the available data, and in Fig. 1 we show a selection of the images we used.

\subsection{Ultraviolet}

To study the continuum UV emission from the young stars of the Tadpole galaxy, we used data from the GALaxy Evolution eXplorer mission GALEX. GALEX is a space telescope providing images in two different ultraviolet bands: the far-UV (FUV), covering the wavelength range from $1350 \AA$ to $1750 \AA$, and the near-UV (NUV), covering the range from $1750 \AA$ to $2750 \AA$ (Martin et al. 2005).

UGC 10214 was observed in the Deep Imaging Survey (DIS) in both bands. We retrieved the calibrated maps from the archive and subtracted the background using the corresponding maps from the archive. We adopted a calibration error of $10 \%$ for both images (Morrissey et al. 2007).

\subsection{Optical}

\subsubsection{Sloan}

We retrieved images of UGC 10214 from the Sloan Digital Sky Survey (SDSS; York et al. 2000) in the $u^{\prime}, g^{\prime}, r^{\prime}, i^{\prime}$, and $z^{\prime}$ bands from the SDSS 9th Data Release (July 2012). We adopted a calibration error of $1 \%$ for the $g^{\prime}, r^{\prime}, i^{\prime}$, and $z^{\prime}$ bands, and $2 \%$ for the $u^{\prime}$ band (Padmanabhan et al. 2008).

\subsection{2. $\mathrm{H} \alpha$}

The study of the ionised gas was performed using the narrowband $\mathrm{H} \alpha$ image from Knierman et al. (in prep.). Observations to obtain $\mathrm{H} \alpha$ images were taken with the Loral 2K CCD imager at the Lennon $1.8 \mathrm{~m}$ Vatican Advanced Technology Telescope (VATT) on Mount Graham, Arizona, on 2004 May 22. This imager has a 6.4 field of view with 0.'42 per pixel. Narrow-band $\mathrm{H} \alpha$ images were obtained at the VATT with an $88 \mathrm{~mm}$ Andover three-cavity interference filter centered at $6780 \AA$. The integration time for each of the six $\mathrm{H} \alpha$ images was $1200 \mathrm{~s}$. To subtract continuum emission, this field was also observed with a KronCousins R filter using integration times of $3 \times 300$ s. Images were reduced using standard IRAF $^{1}$ tasks.

To create images with only the emission lines, a scaled R-band image was subtracted from the narrow-band image after

\footnotetext{
1 IRAF is distributed by the National Optical Astronomy Observatory, which is operated by the Association of Universities for Research in Astronomy, Inc., under cooperative agreement with the National Sci-
} ence Foundation. 

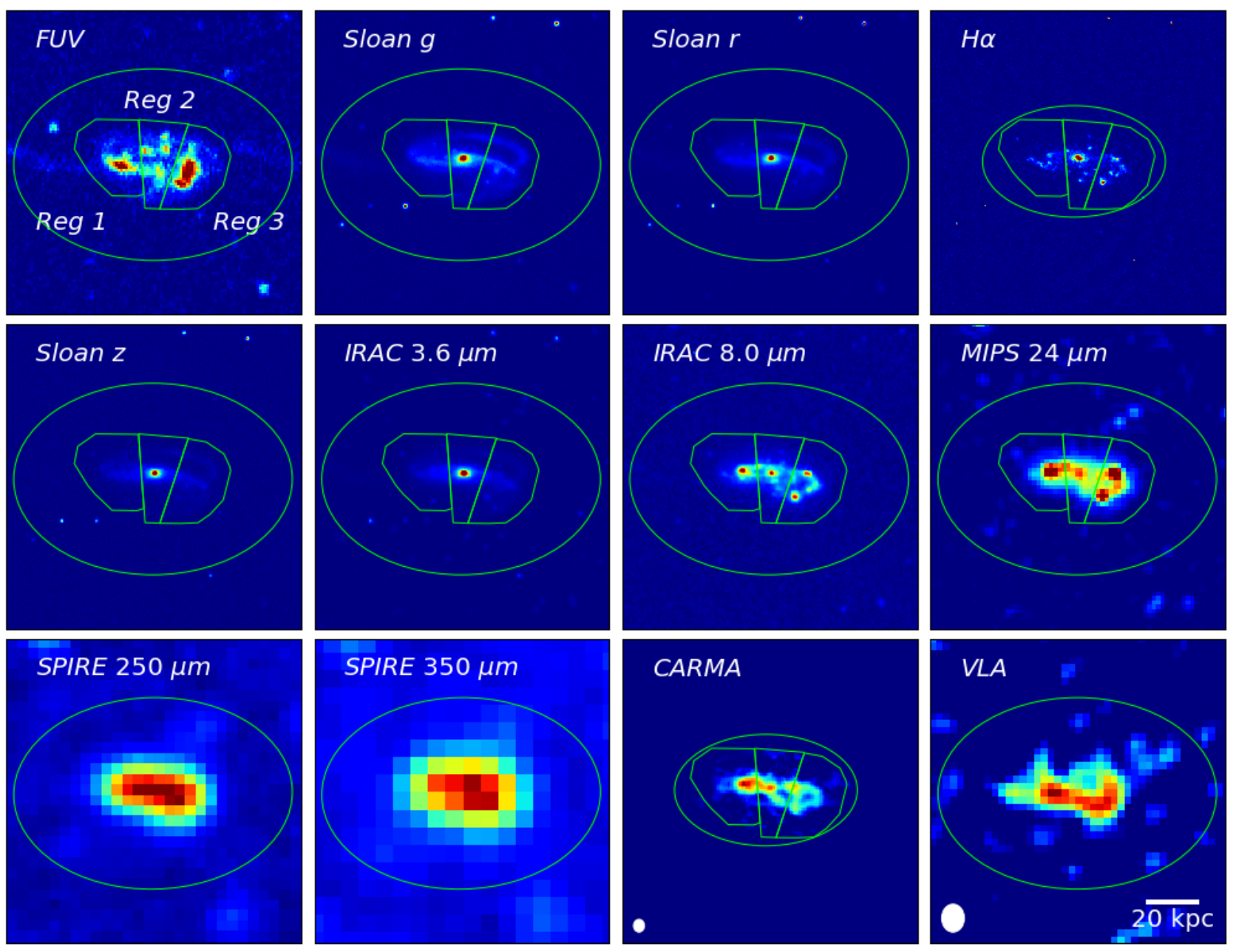

Fig. 1. Representative selection of the images used in our study, together with the apertures used for the three regions (the polygonal shapes) and the disc (the elliptical shape). The elliptical apertures are selected in order to ensure full coverage of the emission of the whole disc. The apertures for the regions in the disc cover three different star-forming regions that are observed in $\mathrm{H} \alpha$ and UV images. The field of view of each image is $170^{\prime \prime} \times 176^{\prime \prime}$. The CARMA and VLA maps show the velocity-integrated intensities. The white ellipse (lower left corner) in the CARMA and VLA images represents the beam size for each image.

alignment using foreground stars. To determine an initial scaling factor, the ratio of integration time for individual frames $(1200 \mathrm{~s} / 300 \mathrm{~s}=4)$ was multiplied by the ratio of filter widths (81 $\AA / 1186.35 \AA=0.068)$. This initial scaling factor then equals 0.272 . We next determined the scaling factor by performing photometry of non-saturated stars in the $\mathrm{H} \alpha$ and $R$-band images. By plotting the instrumental flux of both filters against each other, we determined a linear fit of $f\left(\mathrm{H} \alpha_{\text {inst }}\right)=0.256 \times f\left(R_{\text {inst }}\right)+185.79$ with a correlation coefficient of $r=0.9965$. The slope of this fit gives the appropriate scaling factor between the $R$ and $\mathrm{H} \alpha$ images, which we adopted.

For calibration of the $\mathrm{H} \alpha$ flux, spectrophotometric standard stars from the Oke (1990) catalogue were observed. Aperture photometry of these standards was compared to their absolute magnitudes. Absolute magnitudes for each spectrophotometric standard star were calculated by integrating their SED over the filter response function. We used a standard atmospheric extinction coefficient of 0.08 mag airmass ${ }^{-1}$. Zero-points were calculated by comparing the absolute magnitude in each filter with the instrumental magnitude from aperture photometry. For each night, the zero-points from all standards (in this case, four) were averaged. These zero-points had a standard deviation of $0.016 \mathrm{mag}$. Finally, we removed the contamination from the
[NII] doublet at $6548,6583 \AA$ in the $\mathrm{H} \alpha$ filter, adopting a ratio of $[\mathrm{NII}] / \mathrm{H} \alpha=0.094$ (from Tran et al. 2003).

\subsection{Infrared}

\subsubsection{Spitzer}

To carry out the study of the galaxy in the near- and midinfrared (NIR and MIR), we used a set of images obtained by the Spitzer Space Telescope, consisting of images at $3.6 \mu \mathrm{m}$, $4.5 \mu \mathrm{m}, 5.8 \mu \mathrm{m}$, and $8.0 \mu \mathrm{m}$ taken by the Infrared Array Camera (IRAC; Fazio et al. 2004), as well as an image at $24 \mu \mathrm{m}$ from the Multiband Imaging Photometer (MIPS; Rieke et al. 2004) (project ID: 185, IP C. Londsdale). No other images from the MIPS were available. The images were reduced with the supermoscaic pipeline, and no further data reduction was necessary. The $3.6 \mu \mathrm{m}$ and $4.5 \mu \mathrm{m}$ images mainly provide information about the emission of the oldest star population of the galaxy, the $5.8 \mu \mathrm{m}$ and $8.0 \mu \mathrm{m}$ images inform about the emission from the polycyclic aromatic hydrocarbons (PAH), and the $24 \mu \mathrm{m}$ image show the emission from the hot dust. For all these images we adopted a calibration error of 10\% (Fazio et al. 2004 for IRAC and Rieke et al. 2004 for MIPS). 
Table 1. Summary of the images and data.

\begin{tabular}{|c|c|c|c|c|c|}
\hline Telescope & $\begin{array}{c}\text { Instrument/ } \\
\text { filter/line }\end{array}$ & $\begin{array}{c}\lambda_{0}(\mu \mathrm{m}) \\
\text { or } v_{0}(\mathrm{GHz})\end{array}$ & $F W H M$ & $\begin{array}{l}\triangle_{\mathrm{cal}} \\
(\%)\end{array}$ & $\begin{array}{l}\text { rms noise } \\
\text { of images }\end{array}$ \\
\hline GALEX & FUV & $0.154 \mu \mathrm{m}$ & 3.' 82 & 10 & $2.4 \times 10^{-5} \mathrm{mJy}$ \\
\hline GALEX & NUV & $0.232 \mu \mathrm{m}$ & 5.40 & 10 & $5 \times 10^{-5} \mathrm{mJy}$ \\
\hline Sloan & $u^{\prime}$ & $0.359 \mu \mathrm{m}$ & 1.68 & 2 & $3 \times 10^{-4} \mathrm{mJy}$ \\
\hline Sloan & $g^{\prime}$ & $0.481 \mu \mathrm{m}$ & $1 . \prime 70$ & 1 & $1.1 \times 10^{-3} \mathrm{mJy}$ \\
\hline Sloan & $r^{\prime}$ & $0.623 \mu \mathrm{m}$ & 1."31 & 1 & $1.6 \times 10^{-3} \mathrm{mJy}$ \\
\hline Sloan & $i^{\prime}$ & $0.764 \mu \mathrm{m}$ & $1^{\prime \prime} .24$ & 1 & $2.3 \times 10^{-3} \mathrm{mJy}$ \\
\hline Sloan & $z^{\prime}$ & $0.906 \mu \mathrm{m}$ & 1.! 32 & 1 & $2.5 \times 10^{-3} \mathrm{mJy}$ \\
\hline VATT & $\mathrm{H} \alpha$ & $0.677 \mu \mathrm{m}$ & 0.80 & 3 & $5 \times 10^{-17} \mathrm{erg} \mathrm{cm}^{-2} \mathrm{~s}^{-1}$ \\
\hline Spitzer & IRAC & $3.58 \mu \mathrm{m}$ & 2.16 & 10 & $1.8 \times 10^{-3} \mathrm{mJy}$ \\
\hline Spitzer & IRAC & $4.52 \mu \mathrm{m}$ & $2 . \prime 14$ & 10 & $1.2 \times 10^{-3} \mathrm{mJy}$ \\
\hline Spitzer & IRAC & $5.72 \mu \mathrm{m}$ & $1 . \prime 83$ & 10 & $2.4 \times 10^{-3} \mathrm{mJy}$ \\
\hline Spitzer & IRAC & $7.90 \mu \mathrm{m}$ & $2 . \prime 13$ & 10 & $1.4 \times 10^{-3} \mathrm{mJy}$ \\
\hline Spitzer & MIPS & $23.7 \mu \mathrm{m}$ & $6 . \prime 01$ & 10 & $6 \times 10^{-3} \mathrm{mJy}$ \\
\hline Herschel & SPIRE & $250 \mu \mathrm{m}$ & 19.05 & 15 & $0.6 \mathrm{mJy}$ \\
\hline Herschel & SPIRE & $350 \mu \mathrm{m}$ & $27{ }^{\prime \prime} 18$ & 15 & $0.7 \mathrm{mJy}$ \\
\hline Herschel & SPIRE & $500 \mu \mathrm{m}$ & $45^{\prime \prime} .21$ & 15 & $0.7 \mathrm{mJy}$ \\
\hline CARMA & $\mathrm{CO}(1-0)$ & $111.8 \mathrm{GHz}$ & $6 . .2 \times 7 . ! 3$ & 10 & $2 \mathrm{mJy}$ beam $^{-1}$ \\
\hline IRAM $30 \mathrm{~m}$ & $\mathrm{CO}(1-0)$ & $111.8 \mathrm{GHz}$ & $22^{\prime \prime}$ & 15 & - \\
\hline IRAM $30 \mathrm{~m}$ & $\mathrm{CO}(2-1)$ & $223.6 \mathrm{GHz}$ & $11^{\prime \prime}$ & 25 & - \\
\hline VLA & HI & $1.45 \mathrm{GHz}$ & $13^{\prime \prime}-16^{\prime \prime}$ & 3 & $0.09-0.10 \mathrm{mJy}$ beam $^{-1}$ \\
\hline
\end{tabular}

\subsubsection{Herschel}

To further study the dust emission of the Tadpole galaxy, we used images obtained by the Spectral and Photometric Imaging Receiver (SPIRE; Griffin et al. 2010) on board the Herschel Space Telescope. Images at all three SPIRE bands $(250 \mu \mathrm{m}$, $350 \mu \mathrm{m}$, and $500 \mu \mathrm{m})$ are available in the Herschel archive. The data were observed in SpirePacsParallel mode (project ID: KPGT_soliver_1). The PACS images are not useful because UGC 10124 is too close to the image edge. We retrieved SPIRE level-3 products, which are mosaics obtained by merging all or a subset of contiguous observations. No further data reduction was necessary. Following the instrument handbook ${ }^{2}$, we adopted a calibration error of $15 \%$.

\subsection{Millimeter data}

We carried out two complementary sets of CO observations. The disc of UGC 10214 was observed with the interferometer Combined Array for Research in Millimeter-wave Astronomy (CARMA) at a spatial resolution of $\sim 7^{\prime \prime}$ and a velocity resolution of $81 \mathrm{~km} \mathrm{~s}^{-1}$. In addition, a fully sampled map was obtained with the $30 \mathrm{~m}$ telescope at Pico Veleta of the Institut de Radioastronomie Milimétrique (IRAM). This map has a coarse spatial resolution $\left(22^{\prime \prime}\right)$, but a narrower velocity resolution $\left(\sim 2.5 \mathrm{~km} \mathrm{~s}^{-1}\right)$. The velocities of both data sets were adapted to the definition of the VLA data (optical convention for the transformation of frequency to velocity and heliocentric reference system).

\subsubsection{CARMA}

We observed the Tadpole galaxy in the ${ }^{12} \mathrm{CO}(1-0) 115 \mathrm{GHz}$ transition with CARMA in a nine-point mosaic that covered its head

\footnotetext{
2 http://herschel.esac.esa.int/Docs/SPIRE/spire_ handbook.pdf
}

and tail structure (project code c0269). CARMA was a heterogeneous array that consisted of six antennas with $10.4 \mathrm{~m}$ diameter and nine antennas with $6.1 \mathrm{~m}$ diameter. We obtained data in the D and $\mathrm{E}$ antenna configurations, covering baselines between 8 and $150 \mathrm{~m}$. Twelve D-configuration tracks were taken between 2008 July 20 and 2008 August 29 and nine E-configuration tracks between 2008 September 18 and 2008 October 5, with on-source time variations between 1 and $5 \mathrm{~h}$.

The correlation was set up to cover $468.75 \mathrm{MHz}$ spread over 15 channels, $31.25 \mathrm{MHz}$ wide, using Doppler corrections for the Tadpole with a redshift of $z=0.031358$ relative to the rest frequency of the $\mathrm{CO}$ line at $115.27120 \mathrm{GHz}$. This corresponds to $81.27 \mathrm{~km} \mathrm{~s}^{-1}$ wide channels over a total bandwidth of $1219 \mathrm{~km} \mathrm{~s}^{-1}$.

All data reduction was performed with the MIRIAD package $^{3}$ (Sault et al. 1995). The data were edited and calibrated using sources $0927+390$ or $3 \mathrm{C} 273$ for bandpass (15 min on source), $1638+573$ for complex gain/phase (with cycles of $20 \mathrm{~min}$ on the Tadpole galaxy and $2.5 \mathrm{~min}$ on the calibrator), and Mars ( $5 \mathrm{~min}$ ) for flux calibration, respectively. All data were combined to produce final mosaicked image cubes using natural weighting and joint deconvolution with a synthesized beam of $7^{\prime \prime} .28 \times 66^{\prime \prime} .23 ; \mathrm{PA}=67.1^{\circ}$. The rms noise in the field of view that is covered by all antenna primary beams of the heterogeneous array amounts to $2 \mathrm{mJy}$. We adopted a calibration error of $10 \%$.

We calculated the mass of the molecular gas from the CARMA CO fluxes as

$\left(\frac{M_{\mathrm{H}_{2}}}{M_{\odot}}\right)=1.05 \times 10^{4} \cdot\left(\frac{S}{\mathrm{Jy} \cdot \mathrm{kms}^{-1}}\right) \cdot\left(\frac{D}{\mathrm{Mpc}}\right)^{2}$,

from Bolatto et al. (2013). This expression is based on a conversion factor of $X_{\mathrm{CO}}=2 \times 10^{20} \mathrm{~cm}^{-2} /\left(\mathrm{K} \mathrm{km} \mathrm{s}^{-2}\right)$ and includes a helium fraction of 1.36 .

\footnotetext{
3 http://www.atnf.csiro.au/computing/software/miriad/
} 
Table 2. $\mathrm{CO}(1-0)$ and $\mathrm{CO}(2-1)$ velocity-integrated intensities from IRAM, and comparison to CARMA data.

\begin{tabular}{|c|c|c|c|}
\hline Position & $\begin{array}{c}I_{\mathrm{CO} 10}{ }^{a} \\
\left(\mathrm{~K} \mathrm{~km} \mathrm{~s}^{-1}\right)\end{array}$ & $\begin{array}{c}I_{\mathrm{CO} 21}{ }^{a} \\
\left(\mathrm{~K} \mathrm{~km} \mathrm{~s}^{-1}\right)\end{array}$ & $\frac{M_{\mathrm{H}_{2}}, \text { CARMA }}{M_{\mathrm{H}_{2}}, \text { IRAM }}$ \\
\hline 1 & $1.5 \pm 0.2$ & $<1.1$ & $1.3 \pm 0.3$ \\
\hline 2 & $2.9 \pm 0.2$ & $2.9 \pm 0.5$ & $0.8 \pm 0.2$ \\
\hline 3 & $2.4 \pm 0.3$ & $1.9 \pm 0.4$ & $0.9 \pm 0.2$ \\
\hline 4 & $2.0 \pm 0.2$ & $1.4 \pm 0.3$ & $1.0 \pm 0.2$ \\
\hline 5 & $3.7 \pm 0.3$ & $<2.1$ & $0.4 \pm 0.1$ \\
\hline 6 & $2.4 \pm 0.2$ & $2.1 \pm 0.3$ & $0.7 \pm 0.2$ \\
\hline 7 & $2.0 \pm 0.2$ & $2.4 \pm 0.5$ & $0.7 \pm 0.1$ \\
\hline 8 & $2.7 \pm 0.3$ & $3.00 \pm 0.45$ & $0.7 \pm 0.1$ \\
\hline
\end{tabular}

Notes. ${ }^{(a)}$ Velocity-integrated $\mathrm{CO}(1-0)$ and $\mathrm{CO}(2-1)$ intensities observed at the positions labelled in Fig. 2 (in $T_{\mathrm{mB}}$ ). ${ }^{(b)}$ Flux ratio between CARMA and IRAM at the individual position, derived as explained in Sect. 4.1.3.

\subsubsection{IRAM $30 \mathrm{~m}$}

We observed the $\mathrm{CO}(1-0)$ and $\mathrm{CO}(2-1)$ lines at their redshifted frequencies $(111.8 \mathrm{GHz}$ and $223.6 \mathrm{GHz}$, respectively) between August and October 2005 with the IRAM 30 m telescope on Pico Veleta at eight positions, offset by $9^{\prime \prime}$ and covering the entire disc of UGC 10214. The map is fully sampled for CO(1-0), but undersampled for $\mathrm{CO}(2-1)$. Dual polarisation receivers were used at both frequencies with the $512 \times 1 \mathrm{MHz}$ filterbanks on the $\mathrm{CO}$ (1-0) line and the $256 \times 4 \mathrm{MHz}$ filterbanks on the $\mathrm{CO}(2-1)$. The observations were made in wobbler-switching mode with a wobbler throw of $200^{\prime \prime}$ in azimuthal direction. Pointing was monitored on nearby quasars every $60-90 \mathrm{~min}$. During the observation period, the weather conditions were generally good (with pointing better than $\left.4^{\prime \prime}\right)$. The average system temperatures were $\sim 200 \mathrm{~K}$ at $115 \mathrm{GHz}$ and $\sim 420 \mathrm{~K}$ at $230 \mathrm{GHz}$ on the $T_{\mathrm{A}}^{\star}$ scale. At $111.8 \mathrm{GHz}(223.6 \mathrm{GHz})$, the IRAM forward efficiency, $F_{\text {eff }}$, was $0.95(0.90)$, the beam efficiency, $B_{\text {eff }}$, was $0.75(0.54)$, and the half-power beam size is $22^{\prime \prime}\left(11^{\prime \prime}\right)$. All CO spectra are presented on the main-beam temperature scale $\left(T_{\mathrm{mb}}\right)$, which is defined as $T_{\mathrm{mb}}=\left(F_{\text {eff }} / B_{\text {eff }}\right) \times T_{\mathrm{A}}^{\star}$.

The data were reduced in the standard way via the CLASS software in the GILDAS package ${ }^{4}$. We first discarded poor scans and then subtracted a constant or linear baseline. We then averaged the spectra over the individual positions.

For each spectrum, we visually determined the zero-level line widths, if detected. The velocity-integrated spectra were calculated by summing the individual channels in between these limits. All positions in $\mathrm{CO}(1-0)$ are detected. For $\mathrm{CO}(2-1)$, two positions are not detected, and we obtained the upper limit as

$I_{\mathrm{CO}}<3 \times \mathrm{rms} \times \sqrt{\delta \mathrm{V} \Delta \mathrm{V}}$,

where $\delta \mathrm{V}$ is the channel width and $\Delta V$ the total line width, for which we adopt the same value as for the detected $\operatorname{CO}(1-0)$ line at this position. In addition to the statistical error of the velocityintegrated line intensities, a typical calibration error of $15 \%$ for $\mathrm{CO}(1-0)$ and $25 \%$ for $\mathrm{CO}(2-1)$ has to be taken into account.

In Fig. 2 we show the positions observed by the IRAM $30 \mathrm{~m}$ telescope, overlaid on the CO map of CARMA, together with the $\mathrm{CO}(1-0)$ and $\mathrm{CO}(2-1)$ spectra. In Table 2 the velocity-integrated intensities for the various positions are listed.

\footnotetext{
4 http://www.iram.fr/IRAMFR/GILDAS
}

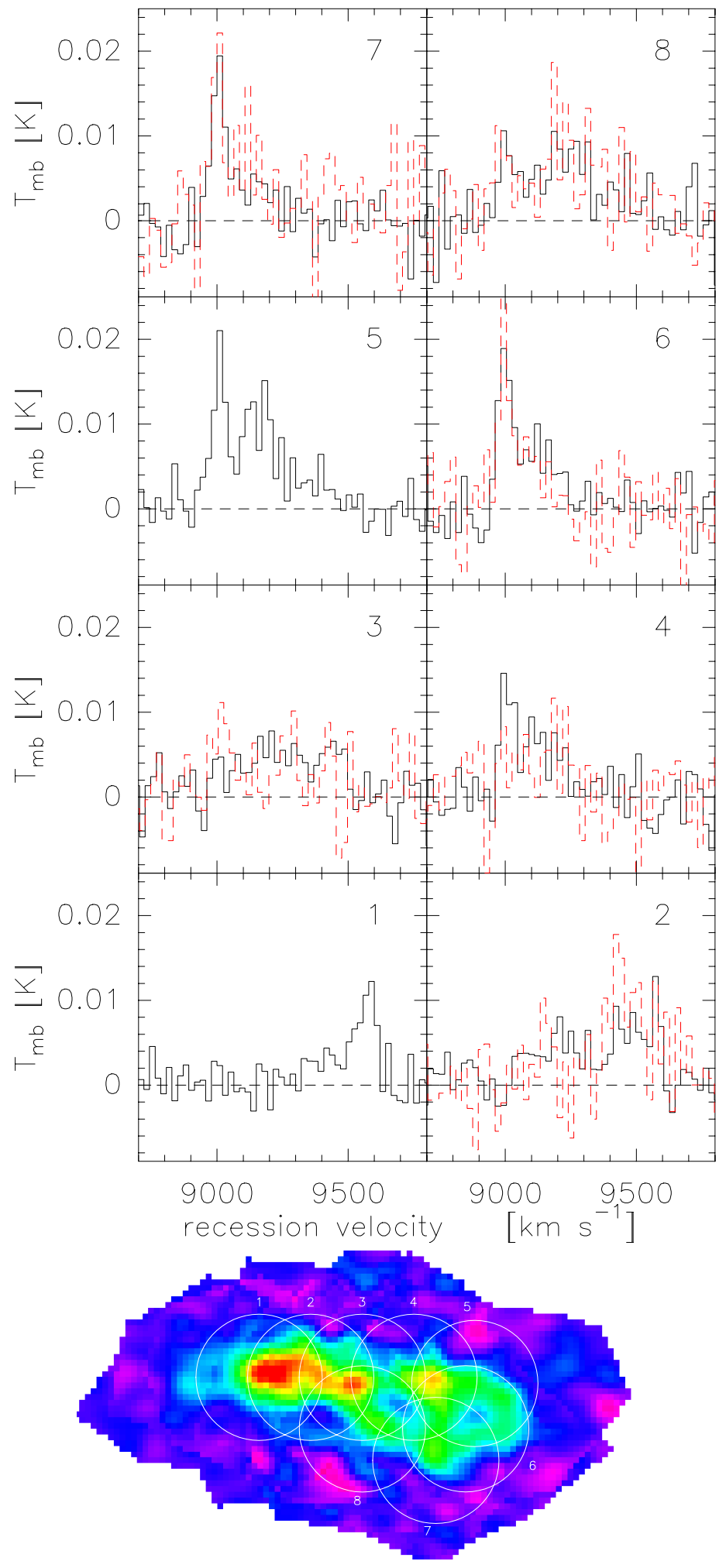

Fig. 2. Upper panel: $\mathrm{CO}(1-0)$ (black, full line) and $\mathrm{CO}(2-1)$ (red, dashed line) spectra observed with the IRAM $30 \mathrm{~m}$ telescope. Only detected spectra are shown. Lower panel: observed positions are indicated on a CARMA CO(1-0) map.

We calculated the molecular gas mass for the IRAM data from the $\mathrm{CO}(1-0)$ velocity-integrated emission using the same value for $X_{\mathrm{CO}}=N\left(\mathrm{H}_{2}\right) / I_{\mathrm{CO}(1-0)}=2 \times 10^{20} \mathrm{~cm}^{-2} /\left(\mathrm{K} \mathrm{km} \mathrm{s}^{-1}\right)$ as for the CARMA data and also including a helium fraction of 1.36 , 
$M_{\mathrm{H}_{2}}=1.36 \cdot I_{\mathrm{CO}(1-0)} \frac{N\left(\mathrm{H}_{2}\right)}{I_{\mathrm{CO}(1-0)}} D^{2} \cdot \Omega \cdot 2 m_{\mathrm{H}}$,

$$
\left(\frac{M_{\mathrm{H}_{2}}}{M_{\odot}}\right)=102 \times 10^{5}\left(\frac{\Omega}{\operatorname{arcsec}^{2}}\right)\left(\frac{I_{\mathrm{CO}(1-0)}}{\mathrm{K} \cdot \mathrm{km} \mathrm{s}^{-1}}\right)\left(\frac{D}{\mathrm{Mpc}}\right)^{2} .
$$

Here, $\Omega$ is the area covered by the observations (i.e. for a single pointing with a Gaussian beam $\Omega=1.13 \theta^{2}$, where $\theta$ is the half-power beam width, HPBW), $D$ is the distance, and $2 m_{\mathrm{H}}$ is the mass of an $\mathrm{H}_{2}$ molecule.

\subsection{Radio (HI data)}

The VLA observations (project code AH841) were conducted on 2004 March 1 with 25 available antennas in the C-array configuration (maximum baseline of $3.4 \mathrm{~km}$ ). At $21 \mathrm{~cm}$, this provides angular resolutions (FWHM of the synthesised beam) of $\sim 13^{\prime \prime}-16^{\prime \prime}$ (depending on weighting, see below), which represents a factor of 2-3 linear improvement over the $36^{\prime \prime} \times 26^{\prime \prime}$ resolution of the Westerbork Radio Synthesis Telescope (WSRT) HI observations of Briggs et al. (2001). The correlator set-up was chosen considering the HI observations of Briggs et al. (2001), which showed the entire HI emission in UGC 10214 to span a total of $900 \mathrm{~km} \mathrm{~s}^{-1}$ (velocities from 8950 to $9850 \mathrm{~km} \mathrm{~s}^{-1}$, using heliocentric velocities and the optical convention to transform frequency shifts into velocity throughout). At that time, the VLA correlator modes could either cover a wide velocity range at very low spectral resolution or a narrower velocity range at higher spectral resolution. As a compromise, we elected to use the 4IF mode, which allowed us to use different bandwidth codes for each IF pair. Both IF pairs were tuned to a central frequency corresponding to the peak tail emission $\left(9420 \mathrm{~km} \mathrm{~s}^{-1}\right)$. The first IF pair used bandwidth code 5 to provide 63 channels across a $1.56 \mathrm{MHz}$ bandwidth (velocity coverage $\sim 9250-9590 \mathrm{~km} \mathrm{~s}^{-1}$ with a channel spacing of $5.5 \mathrm{~km} \mathrm{~s}^{-1}$ ). These data are referred to as the narrow-band data. The second IF pair used bandwidth code 3 to provide 15 channels across a $6.25 \mathrm{MHz}$ bandwidth (velocity coverage $\sim 8806-10034 \mathrm{~km} \mathrm{~s}^{-1}$ with a channel spacing of $88 \mathrm{~km} \mathrm{~s}^{-1}$ ). These data are referred to as the wide-band data. The narrow-band data provide a higher velocity resolution to resolve the kinematics of the tidal gas (measured velocity dispersion of $17 \mathrm{~km} \mathrm{~s}^{-1}$ from Briggs et al. 2001), but do not measure the full extent of the disc gas or intruding dwarf galaxy (VV 29c), while the wide-band data provide sufficient bandwidth to measure all $\mathrm{HI}$ associated with the system with line-free channels on either end, but at a very coarse velocity resolution. We here only use the wide-band data of the disc of UGC 10214. The wide- and narrow-band data of the tidal tail will be presented and analysed in Knierman et al. (in prep.).

The flux calibrator 3C286 $(1331+305)$ was observed during $15 \mathrm{~min}$ at the start of the observation, followed by pairs of observations on the phase calibrator $(1634+627,10$ min per visit) and UGC 10214 (45 min per visit). This phase calibrator was bright enough at $21 \mathrm{~cm}(5 \mathrm{Jy})$ to be also used as the bandpass calibrator. The total observation spanned $9 \mathrm{~h}$, with $6.5 \mathrm{~h}$ on-source.

The data were reduced using the Astronomical Image Processing System (AIPS). The continuum was subtracted based on the line-free channels using the AIPS task UVLIN. This is a visibility-domain technique that removes a linear fit to the line-free channels from each visibility. The data were imaged with various "robust" weighting factors (Briggs 1995), yielding FWHM beam sizes between $\sim 13^{\prime \prime}$ and $16^{\prime \prime}$ and rms noises between 0.09 and $0.10 \mathrm{mJy}$ beam $^{-1}$. The resulting images are

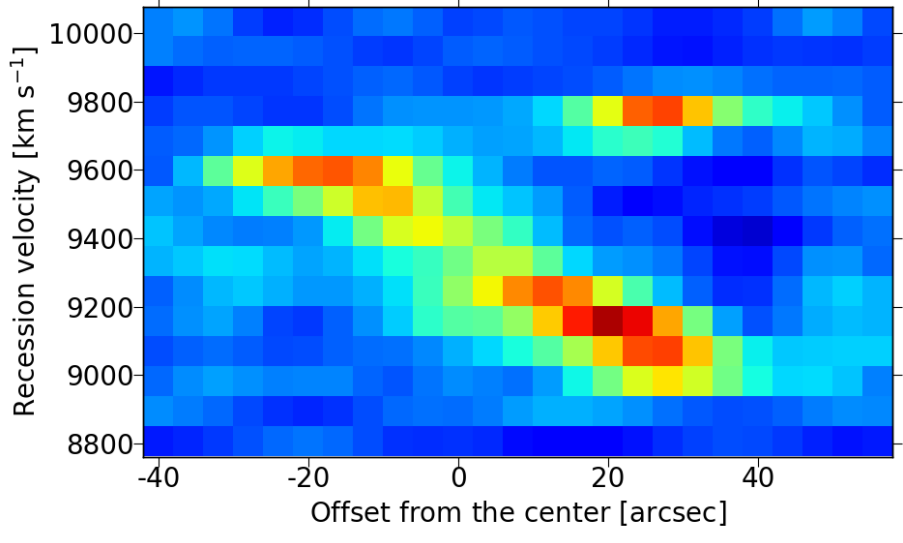

Fig. 3. Pv diagram of the HI in UGC 10214 (inclined feature towards the east) and VV 29c (compact feature at $\sim 9750 \mathrm{~km} \mathrm{~s}^{-1}$ ), taken along the major axis of UGC 10214, with a slice width of $8^{\prime \prime}$ and a position angle of $80^{\circ}$. Negative offsets are to the east and positive offsets to the west.

three-dimensional cubes of $\mathrm{HI}$ intensity (in Jy beam ${ }^{-1}$ ) as a function of RA and Dec; and velocity. We used the data cube with a "robust" weighting factor of +1 , which provides the coarsest spatial resolution $\left(15^{\prime \prime} .5 \times 16^{\prime \prime} 0\right)$, but reflects most reliably the total flux and has a higher signal-to-noise ratio. We inspected the robust -1 map with a higher resolution $\left(122^{\prime \prime} 6 \times 12\right.$.' 9$)$ for the presence of new features that were not visible in the robust +1 map, but did not find any.

These data include both the emission from UGC 10214 and from the background dwarf galaxy VV 29c. The velocities of the HI emission from VV 29c are offset from the velocities of UGC 10214 at this position, so that both objects can be clearly distinguished in a position-velocity (pv) diagram (Fig. 3). We separated the emission from UGC 10214 and VV 29c into individual data cubes by masking the channels with velocities between 9630 and $9800 \mathrm{~km} \mathrm{~s}^{-1}$ that were in the area of VV 29c (RA between 16:05:3.9 and 16:05:58.3 and Dec between 55:25:00 and 55:25:52). The resulting data cube represents the HI emission from UGC 10214 alone. The emission that was masked out in the first step provides the emission of VV 29c.

We derived the total flux for UGC 10214 and VV 29c by summing all channels from the corresponding data cube over the entire region of each galaxy. The resulting spectrum was then integrated over velocity in the range where the line is visible (8850-9800 $\mathrm{km} \mathrm{s}^{-1}$ for UGC 10214 and 9630 and $9800 \mathrm{~km} \mathrm{~s}^{-1}$ for VV 29c). The error was calculated by the quadratic sum of the error due to the per-channel rms noise and of the calibration error of $\sim 3 \%{ }^{5}$.

We calculated the atomic gas mass from the total flux, $S_{\mathrm{HI}}$, as

$\left(\frac{M_{\mathrm{HI}}}{M_{\odot}}\right)=1.36 \cdot 2.36 \times 10^{5} \cdot\left(\frac{S_{\mathrm{HI}}}{\mathrm{Jy} \cdot \mathrm{kms}^{-1}}\right) \cdot\left(\frac{D}{\mathrm{Mpc}}\right)^{2}$,

where the factor 2.36 is the standard value to calculate the atomic gas mass (e.g. Roberts \& Haynes 1994; Rohlfs \& Wilson 1996) and the factor 1.36 takes into account the contribution from helium in order to be consistent with the molecular gas mass calculation.

\footnotetext{
5 https://science.nrao.edu/facilities/vla/docs/ manuals/oss2013A/performance/cal-fluxscale
} 


\section{Method}

\subsection{Aperture photometry}

Before carrying out the aperture photometry, we set all the images, except for the SPIRE images and the radio data, to the same spatial resolution and pixel size. We chose the resolution $\left(6^{\prime \prime}\right.$, corresponding to $4 \mathrm{kpc}$ at the distance of UGC 10214) and the pixel size (2.'45) of the MIPS $24 \mu \mathrm{m}$ image as reference. We converted the other images into the MIPS $24 \mu \mathrm{m}$ pointspread function using the set of kernels provided by Aniano et al. $(2011)^{6}$. Then we regridded the images to the MIPS $24 \mu \mathrm{m}$ pixel size using the IRAF task WREGISTER, and at the same time matched the field of view and orientation. The SPIRE images and the VLA radio data have a coarser resolution than the MIPS $24 \mu \mathrm{m}$ map. We therefore excluded these images from any spatially resolved analysis and only measured the total fluxes. The CO CARMA map has a spatial resolution $\left(6{ }^{\prime \prime} 23 \times 77^{\prime \prime} 23\right)$, which is slightly higher than that of the MIPS $24 \mu \mathrm{m}$ map. We neglected this small difference and used the regridded (but unconvolved) CO map for our analysis.

We carried out aperture photometry within an ellipse encompassing the entire galaxy disc at all wavelengths in order to obtain the total fluxes. For most images, except for $\mathrm{H} \alpha$ and CARMA, this ellipse has a major semi-axis of $80^{\prime \prime}$ and a minor semi-axis of $55^{\prime \prime}$. The size was chosen to be large enough to cover the complete emission of the disc at all wavelengths. For $\mathrm{H} \alpha$, we used a smaller aperture $\left(522^{\prime \prime} 5 \times 32^{\prime \prime} .0\right)$ that included the entire $\mathrm{H} \alpha$ emission, but was small enough to avoid a region to the west with some weak artefacts.

In addition, we performed aperture photometry for three regions in the disc that are characterised by different star formation properties (see Fig. 1 for the contour of the apertures) to compare them. We separated the regions based on the distribution of FUV and $\mathrm{H} \alpha$ emissions: regions 1 and 3 contain the maxima in the FUV, but only weak $\mathrm{H} \alpha$ emission, while region 2 in the centre shows the opposite behaviour (the SPIRE and VLA data were excluded from this analysis). The total area covered by the three regions is smaller than the elliptical aperture used to measure the emission of the entire disc.

Following the IRAC handbook, we applied aperture correction factors to the Spitzer IRAC images with values of 0.92 , $0.94,0.80$, and 0.77 for the IRAC bands $1-4$ for all galaxies and $0.93,0.95,0.85$, and 0.80 for the IRAC bands $1-4$ for the smaller subregions. All fluxes were corrected for Galactic extinction by adopting $A_{V}=0.025 \mathrm{mag}$ from the Nasa Extragalactic Database. This was calculated following the recalibration of Schlafly \& Finkbeiner (2011) of the Schlegel et al. (1998) dust maps. The recalibration to other wavelengths was made following the Cardelli et al. (1989) extinction law using the calculator by Dough Welch ${ }^{7}$.

Photometry was performed using different options of the Python Astropy package ${ }^{8}$. In particular, we used the affiliated package Photutils ${ }^{9}$ to carry out the photometry with the elliptical apertures. For the photometry of regions 1-3, we defined irregular polygonal masks with the package Astropy for each region and determined the total fluxes within the mask using the NumPy package (van der Walt et al. 2011). The background was measured using the median value of the pixels surrounding the galaxy disc that are not covered by the polygonal masks. The

6 http://www . astro.princeton. edu/ ganiano/Kernels.html

http: //www . dougwelch.org/Acurve.html

Astropy Collaboration 2013, 2018.

9 Bradley et al. 2017. resulting fluxes are listed in Table 3 . The errors for the optical and infrared images were determined using the following expression:

$\Delta F=\sqrt{F^{2} \frac{\Delta K^{2}}{K^{2}}+\sigma^{2} N_{\mathrm{A}}+\sigma^{2} \frac{N_{\mathrm{A}}^{2}}{N_{\mathrm{S}}}}$,

where $K$ and $\Delta K$ are the calibration for each image and its error, $F$ is the flux measured within the aperture, $\sigma$ is the root-meansquare noise within the background aperture, and $N_{\mathrm{A}}$ and $N_{\mathrm{S}}$ are the numbers of pixels in the galaxy aperture and in the aperture used to measure the background emission, respectively. The first term of the sum gives the error associated with the instrument calibration, the second is the error related to the aperture employed, and the third term is the error that is due to the background subtraction in each image.

The error for the photometry obtained with the CARMA image, for which the background is zero, and with the FUV map, which is already background-subtracted, was derived from the first two terms in the quadratic sum alone.

\subsection{SED modelling with CIGALE}

The SED modelling was carried out with CIGALE (Noll et al. 2009; Boquien et al. 2019). CIGALE is based on an energy balance approach, the energy absorbed by dust at short wavelengths is re-emitted self-consistently. The physical properties and the uncertainties are estimated from the likelihood-weighted means and standard deviations over a grid of models.

The code is highly modular to allow for a flexible modelling. In the present case, the star formation history was modelled through two decaying exponentials, the first modelling the long-term star formation, and the second the latest episode of star formation. Each exponential has an independent age $\left(t_{1}\right.$ and $\left.t_{2}\right)$ and timescale $\left(\tau_{1}\right.$ and $\left.\tau_{2}\right)$, and they are linked through the burst fraction $f$, giving the total fraction of stars formed in the second exponential relative to the total mass of stars ever formed. The stellar emission was computed using the model by Bruzual \& Charlot (2003). The nebular emission, which includes recombination lines as well as continuum originating from free-free, free-bound, and two-photon emission, is based on the number of ionising photons (assuming that all ionising photons ionise the gas) and templates improved from Inoue (2011) computed from CLOUDY 08.00 (Ferland et al. 1998). The attenuation was based on a power-law modified star-burst curve (Calzetti et al. 2000), allowing for a differential reddening between stars older and younger than $10 \mathrm{Myr}$ and with an optional UV bump at $217.5 \mathrm{~nm}$. Finally, dust emission was modelled using the Dale et al. (2014) templates. In the unique case of determining the dust mass, we only fitted infrared data with the dust templates used by Draine et al. (2014). This yielded a total of 20003760 models.

The run was based on a pre-release of version 0.12 modified to fit the $\mathrm{H} \alpha$ emission in addition to broad-band fluxes. The range of the modelled values we used for the different parameters as well as the best-fit parameters for the entire galaxy are listed in Table 4 (the best-fit values for the individual regions are very similar). The beginning of the long-term star formation (parameter $t_{1}$ ) was fixed to a reasonable value of $12 \mathrm{Gyr}$ in order to reduce the number of free parameters. The metallicities were tested for two values (roughly solar and super-solar) that are plausible for this high-mass galaxy and for which stellar emission models were available from Bruzual \& Charlot (2003). No satisfactory fit was achieved with the super-solar metallicity. 
Table 3. Fluxes for the entire disc and the three disc regions.

\begin{tabular}{|c|c|c|c|c|c|}
\hline $\begin{array}{l}\text { Telescope/Instrument } \\
\text { Band }\end{array}$ & Unit & Total & Flux Reg 1 & Flux Reg 2 & Flux Reg 3 \\
\hline GALEX FUV & mJy & $0.29 \pm 0.03$ & $0.073 \pm 0.007$ & $0.072 \pm 0.007$ & $0.098 \pm 0.010$ \\
\hline GALEX NUV & mJy & $0.53 \pm 0.05$ & $0.13 \pm 0.01$ & $0.13 \pm 0.01$ & $0.17 \pm 0.02$ \\
\hline Sloan $u^{\prime}$ & mJy & $1.57 \pm 0.04$ & $0.44 \pm 0.01$ & $0.54 \pm 0.01$ & $0.52 \pm 0.01$ \\
\hline Sloan $g^{\prime}$ & mJy & $7.10 \pm 0.08$ & $1.93 \pm 0.03$ & $2.71 \pm 0.03$ & $1.99 \pm 0.03$ \\
\hline Sloan $r^{\prime}$ & mJy & $13.63 \pm 0.15$ & $3.60 \pm 0.05$ & $5.81 \pm 0.06$ & $3.45 \pm 0.04$ \\
\hline Sloan $i^{\prime}$ & mJy & $19.2 \pm 0.2$ & $5.01 \pm 0.06$ & $8.66 \pm 0.09$ & $4.67 \pm 0.06$ \\
\hline Sloan $z^{\prime}$ & mJy & $24.22 \pm 0.25 \mathrm{f}$ & $6.23 \pm 0.07$ & $11.6 \pm 0.1$ & $5.78 \pm 0.07$ \\
\hline VATT H $\alpha$ & $10^{-14} \mathrm{erg} \mathrm{cm}^{-2} \mathrm{~s}^{-1}$ & $35.8 \pm 1.1$ & $8.2 \pm 0.3$ & $15.0 \pm 0.5$ & $10.9 \pm 0.3$ \\
\hline IRAC 3.6 & mJy & $18.9 \pm 1.9$ & $4.7 \pm 0.5$ & $9.5 \pm 0.9$ & $4.1 \pm 0.4$ \\
\hline IRAC 4.5 & mJy & $12.2 \pm 1.2$ & $3.1 \pm 0.3$ & $6.0 \pm 0.6$ & $2.8 \pm 0.3$ \\
\hline IRAC 5.8 & mJy & $14.4 \pm 1.4$ & $4.3 \pm 0.4$ & $6.8 \pm 0.7$ & $4.4 \pm 0.4$ \\
\hline IRAC 8.0 & mJy & $26.3 \pm 2.6$ & $8.2 \pm 0.8$ & $10.0 \pm 1.0$ & $9.3 \pm 0.9$ \\
\hline MIPS 24 & mJy & $23.9 \pm 2.4$ & $6.5 \pm 0.7$ & $7.3 \pm 0.7$ & $9.1 \pm 0.9$ \\
\hline SPIRE 250 & mJy & $940 \pm 170$ & - & - & - \\
\hline SPIRE 350 & mJy & $450 \pm 100$ & - & - & - \\
\hline SPIRE 500 & mJy & $156 \pm 40$ & - & - & - \\
\hline CARMA CO & $\mathrm{Jy} \mathrm{km} \mathrm{s}^{-1}$ & $32 \pm 3$ & $10 \pm 1$ & $13 \pm 1$ & $9 \pm 1$ \\
\hline VLA HI & $\mathrm{Jy} \mathrm{km} \mathrm{s}^{-1}$ & $3.8 \pm 0.1$ & - & - & - \\
\hline
\end{tabular}

Notes. The apertures used for the regions are described in Sect. 3.1 and shown in Fig. 1.

Table 4. Modelled values (see text) of the different parameters used for the SED modelling with CIGALE.

\begin{tabular}{lll}
\hline \hline Parameters $^{a}$ & Range of modelled values & Best-fit values $^{b}$ \\
\hline Age of the main population; $t_{1}$ & $12 \mathrm{Gyr}$ & $12 \mathrm{Gyr}$ \\
Age of the last episode of star formation; $t_{2}$ & $10-000 \mathrm{Myr}$ & $11 \pm 20 \mathrm{Myr}$ \\
Timescale of the main population; $\tau_{1}$ & $2-10 \mathrm{Gyr}$ & $2.20 \pm 0.06 \mathrm{Gyr}$ \\
Timescale of the last episode of star formation; $\tau_{2}$ & $10-1000 \mathrm{Myr}$ & $310 \pm 340 \mathrm{Myr}$ \\
Burst fraction; $\log f$ & -4 to -1 & $-3.7 \pm-3.8$ \\
IMF & Chabrier & Chabrier \\
Metallicity; $Z$ & $0.02,0.05$ & 0.02 \\
Ionisation parameter; $\log U$ & -3 & -3 \\
Reddening of populations younger than $10 \mathrm{Myr} ; E(B-V)_{\text {young }}$ & $0.05-0.70 \mathrm{mag}$ & $0.18 \pm 0.03$ \\
Relative reddening of populations older than $10 \mathrm{Myr} ; E(B-V)_{\text {old factor }}$ & $0.25-0.75$ & $0.05 \pm 0.01$ \\
UV bump amplitude & $0.0,1.5,3.0$ & 0 \\
Incident dust radiation field intensity power-law distribution; $\alpha$ & $1.0-4.0$ & 3.0 \\
\hline
\end{tabular}

Notes. ${ }^{(a)}$ See text (Sect. 3.2) for a detailed explanation of the parameters. ${ }^{(b)}$ Best-fit values derived from the total fluxes from Table 3. The results for the individual regions are similar.

The best-fit results for the last episode of SF give a timescale of several $100 \mathrm{Myr}$ and an age of several $10 \mathrm{Myr}$. The stellar mass fraction formed in this last episode is small $(0.02 \%)$, which is not surprising given the high stellar mass of UGC 10214. Table 5 lists the SFRs averaged over 10 and $100 \mathrm{Myr}$ and the stellar masses for the total disc and the individual regions, as well as the total dust masses, which we discuss in more detail in Sect. 4. The best-fit SED for the total galaxy is shown in Fig. 4.

\subsection{Pixel-by-pixel analysis}

In addition to analysing the integrated fluxes for the entire galaxy and the three regions, we compared the SFR, molecular gas mass, and stellar mass on a pixel-by-pixel basis using the FUV, $\mathrm{H} \alpha$, CARMA, and IRAC $3.6 \mu$ m maps. We only took into account the pixels for which all five images had significant fluxes at a $3 \sigma$ level. For this, we measured the mean value and standard deviation outside the main galaxy in all five images and created masks consisting of the pixels with fluxes higher than three times the standard deviation. The final mask is shown in Fig. 5. The size of the mask is mainly restricted by the CO map.

\section{Results}

\subsection{Atomic and molecular gas and dust}

\subsubsection{Atomic and molecular gas mass and distribution}

The molecular gas in the CARMA map shows an asymmetric distribution. In the eastern part (region 1), the molecular gas has an elongated distribution with a peak. The molecular gas surface density is highest in this region. In the middle (region 2) and western part (region 3) lies a double-ring structure of lower intensity (see Fig. 1). The western-most ring is reflected in the SFR, clearly seen in the IRAC $8.0 \mu \mathrm{m}$, MIPS $24 \mu \mathrm{m}$, and $\mathrm{H} \alpha$ images. 
Table 5. Results from the modelling with CIGALE.

\begin{tabular}{lcccc}
\hline \hline Region & $\begin{array}{c}\text { SFR }(10 \mathrm{Myr}) \\
{\left[M_{\odot} \mathrm{yr}^{-1}\right]}\end{array}$ & $\begin{array}{c}\text { SFR }(100 \mathrm{Myr}) \\
{\left[M_{\odot} \mathrm{yr}^{-1}\right]}\end{array}$ & $\begin{array}{c}M_{\star} \\
{\left[10^{10} M_{\odot}\right]}\end{array}$ & $\begin{array}{c}M_{\text {dust }} \\
{\left[10^{8} M_{\odot}\right]}\end{array}$ \\
\hline Disc & $4.8 \pm 0.5$ & $0.77 \pm 0.08$ & $12.8 \pm 0.7$ & $1.7 \pm 0.3$ \\
Region 1 & $1.31 \pm 0.09$ & $0.21 \pm 0.03$ & $3.4 \pm 0.2$ & - \\
Region 2 & $1.29 \pm 0.06$ & $0.26 \pm 0.06$ & $5.9 \pm 0.3$ & - \\
Region 3 & $1.4 \pm 0.2$ & $0.4 \pm 0.2$ & $3.1 \pm 0.5$ & - \\
\hline
\end{tabular}

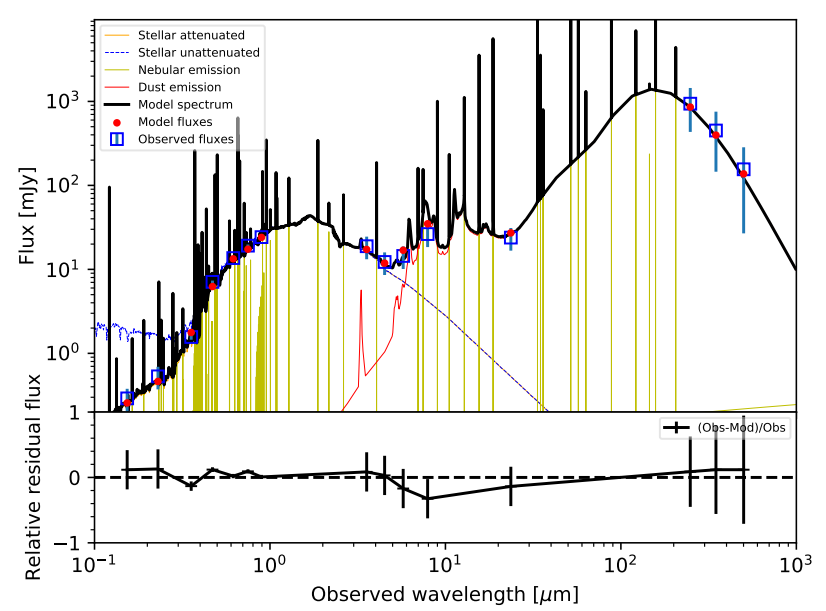

Fig. 4. SED fitting with CIGALE for the disc of UGC 10214.

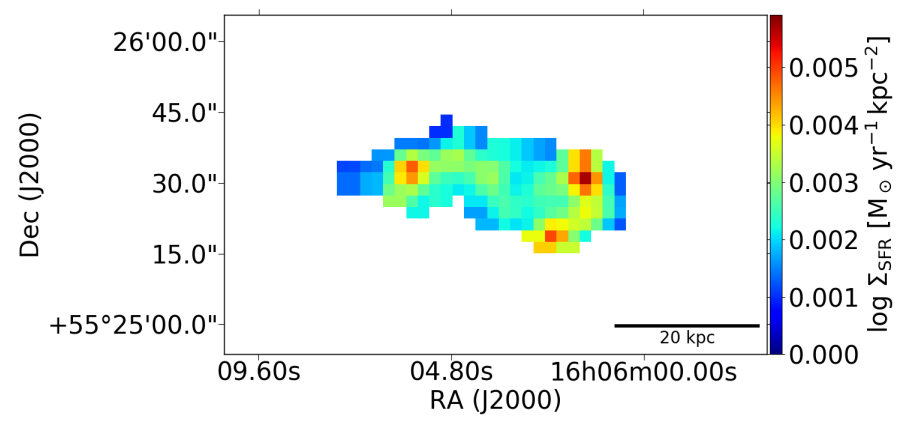

Fig. 5. Final mask used for the pixel-by-pixel analysis, applied for illustration to the SFR map obtained from the dust-corrected FUV emission (see Sect. 4.2).

The atomic gas is much more extended than the molecular gas (Fig. 6). It shows a ridge-like structure from east to west that is offset to the south from the molecular gas. At the western end, it shows an arc-like structure that coincides with the western end of the molecular gas distribution.

The total atomic gas mass, $(2.21 \pm 0.07) \times 10^{10} M_{\odot}$, see Table 6) is a factor 3.7 higher than the total molecular gas mass from CARMA, $(6.0 \pm 0.6) \times 10^{9} M_{\odot}$. This factor is slightly lower when the molecular gas mass from the IRAM single-dish data is used, $(9.0 \pm 1.5) \times 10^{9} M_{\odot}$, corresponding to $M_{\mathrm{HI}} / M_{\mathrm{H}_{2}} \sim 2.5$.

\subsubsection{Kinematics of the atomic and molecular gas}

Figure 7 shows the pv diagram of $\mathrm{HI}$ and of $\mathrm{CO}$ (from CARMA) along the major axis of the disc of UGC 10214. HI and CO both follow a regular velocity distribution, which indicates a rotational disc. The $\mathrm{HI}$ lines are wider and their distributions are more extended towards the western side than those of CO.

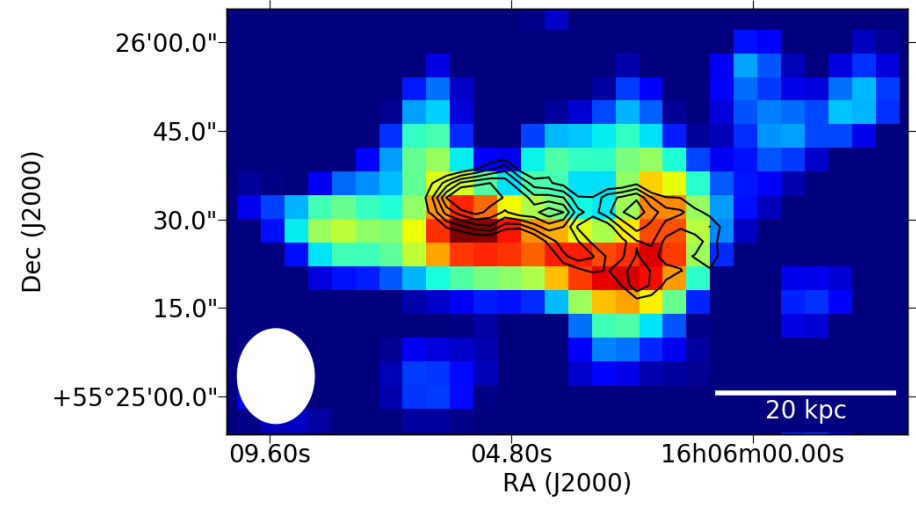

Fig. 6. Distribution of the HI emission of UGC 10214 (colour) and the $\mathrm{CO}(1-0)$ data from CARMA (contours, ranging from 6 to $14 \sigma$ in $2 \sigma$ steps). The ellipse in the lower left corner shows the resolution of the HI data.

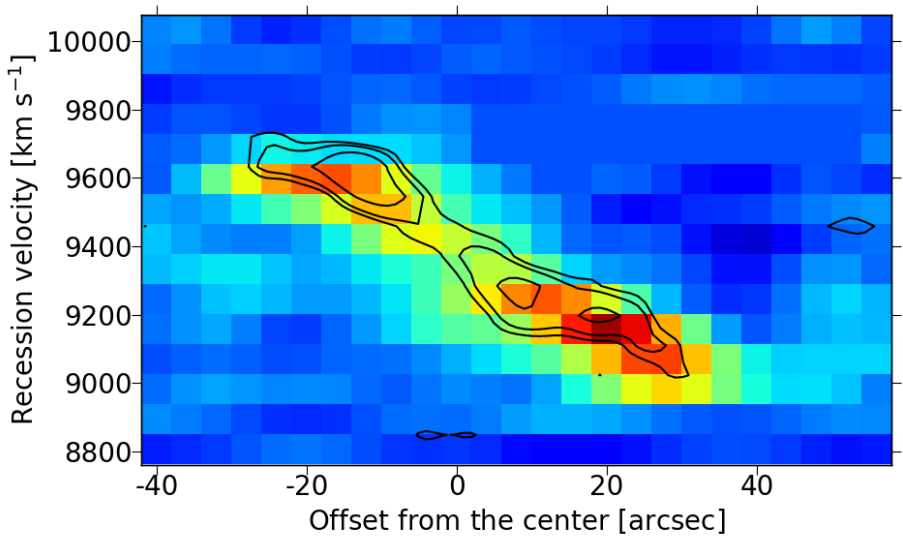

Fig. 7. Pv diagram of UGC 10214 from the HI data (colour) and the CO(1-0) data from CARMA (contours), taken along the major axis of UGC 10214 as in Fig. 3 with a slice width of 4 " on either side.

The high-velocity resolution of the IRAM observations provides additional information about the kinematics of the gas (Fig. 2). Consistent with the CARMA data, a velocity gradient from the eastern to the western part of the galaxy is visible in the IRAM spectra. In addition, the line-shape changes drastically from a relatively weak single-peak spectrum in the east (position 1) to broader, weaker lines in the middle (positions 2, 3, 4, and 8) to a strong peak with a broader, weaker secondary peak in the west (positions 5, 6, and 7).

Figure 8 shows the $\mathrm{HI}$ and $\mathrm{CO}$ spectra integrated over the entire disc. The HI spectrum extends to slighly higher velocities, which is consistent with a more extended disc. The HI spectrum follows the IRAM spectrum more closely and includes the peak at $9200 \mathrm{~km} \mathrm{~s}^{-1}$. The strong peak at $9000 \mathrm{~km} \mathrm{~s}^{-1}$ visible in the IRAM spectrum would be lower by a factor of about 2 if it 


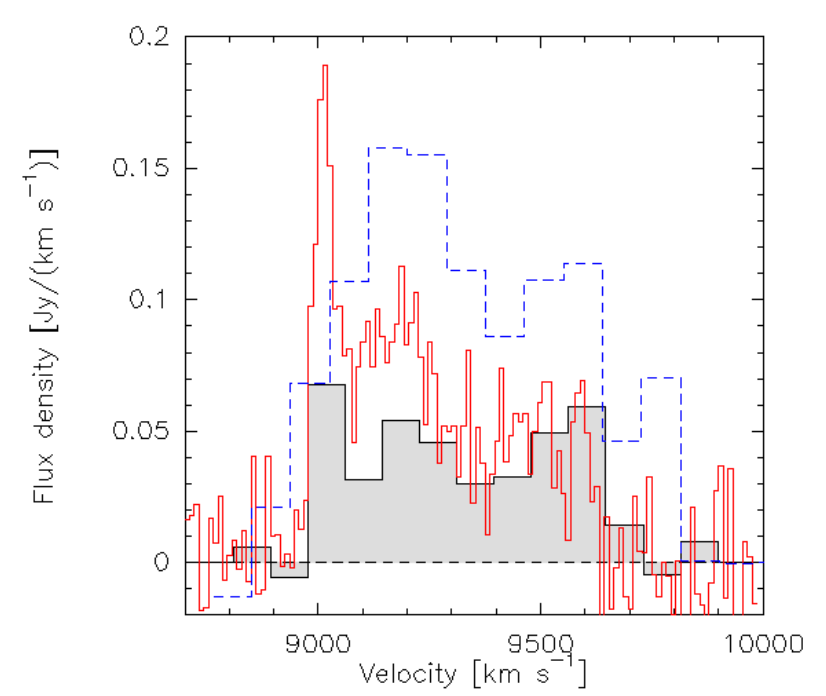

Fig. 8. $\mathrm{CO}(1-0)$ spectra form the IRAM $30 \mathrm{~m}$ telescope (red, continuous line) and from CARMA (grey shaded), together with the HI emission (blue dashed line) integrated over the entire disc of the Tadpole galaxy. The HI spectrum contains only the emission of UGC 10214 (i.e. the emission from VV 29c has been excluded as described in Sect. 2.5). The HI flux is multiplied by a factor of 15 for better visibility.

were smoothed to the velocity resolution of the CARMA and HI data. Taking this decrease into account, it is only slightly higher than the CARMA data. The HI data, on the other hand, show no indication for a strong emission at this velocity.

\subsubsection{Comparison of IRAM and CARMA data}

The total molecular gas mass obtained with the IRAM measurements is $(9 \pm 2) \times 10^{9} M_{\odot}$, a factor of about 1.5 higher than the total flux from CARMA. This indicates that some flux $(\sim 30 \%)$ might be missing in the interferometer measurements.

In Table 2 we list the ratio between the molecular gas masses calculated from the IRAM velocity-integrated intensities (from Eq. (4)) and from the CARMA data. For this comparison we multiplied the velocity-integrated CARMA map (from Fig. 1) with a Gaussian beam of FWHM 20" (in order to convolve from the CARMA resolution to the IRAM FWHM) for each position observed by IRAM and measured the total flux from the resulting image. We then calculated the total molecular gas mass from Eq. (1). The ratio of the CARMA-to-IRAM gas masses spans a range from 0.4 to 1.3 , with the higher values being present in the eastern part of the galaxy and the lower in the western. This indicates that in the western part some flux might be missing from the interferometer measurements.

We compare the total CO spectra, integrated over the entire disc, measured with CARMA and with the IRAM $30 \mathrm{~m}$ in Fig. 8. The total line widths agree well. At $v \sim 9000 \mathrm{~km} \mathrm{~s}^{-1}$, there is a pronounced peak in the IRAM spectrum that is not seen in the CARMA spectrum due to its lower resolution. The missing flux seems to come in part from this peak as well as from the broad velocity feature around $9200 \mathrm{~km} \mathrm{~s}^{-1}$. Both features are emitted in the western part of the galaxy (see Fig. 2).

\subsubsection{Comparison of $I_{\mathrm{CO}(1-0)}$ and $I_{\mathrm{CO}(2-1)}$}

To interpret the ratio of $I_{\mathrm{CO}(2-1)} / I_{\mathrm{CO}(1-0)}$, we have to consider two main parameters: the source distribution, and the opacity. For optically thick thermalised emission with a point-like distribution, we expect a ratio $I_{\mathrm{CO}(2-1)} / I_{\mathrm{CO}(1-0)}=\left(\theta_{\mathrm{CO}(1-0)} /\right.$ $\left.\theta_{\mathrm{CO}(2-1)}\right)^{2}=4$ (with $I_{\mathrm{CO}}$ in $T_{\mathrm{mB}}$ and $\theta$ being the FWHM of the beams). On the other hand, for a uniform source brightness distribution, we expect ratios higher than 1 for optically thin gas and ratios between about 0.6 and 1 for optically thick gas (with excitation temperatures above $5 \mathrm{~K}$ ). Thus, values of $I_{\mathrm{CO}(2-1)} / I_{\mathrm{CO}(1-0)}>1$ indicate a source distribution with an extension smaller than $\theta_{\mathrm{CO}(1-0)}$, while $I_{\mathrm{CO}(2-1)} / I_{\mathrm{CO}(1-0)} \lesssim 0.6$ indicates diffuse subthermally excited gas.

The mean line ratio, averaged over all pointings, is $I_{\mathrm{CO}(2-1)} / I_{\mathrm{CO}(1-0)}=0.8 \pm 0.2$. This value is close to the mean value found by Leroy et al. (2009) from $\operatorname{CO}(2-1)$ and $\mathrm{CO}(1-0)$ maps for nearby galaxies from the SINGS sample $\left(I_{\mathrm{CO}(2-1)} / I_{\mathrm{CO}(1-0)} \sim 0.8\right)$ and those from Braine et al. (1993), who obtained a mean line ratio of $I_{\mathrm{CO}(2-1)} / I_{\mathrm{CO}(1-0)}=0.89 \pm 0.06$ for a sample of nearby spiral galaxies. These two values are, in contrast to ours, corrected for beam-size effects. The ratios of the $I_{\mathrm{CO}(2-1)} / I_{\mathrm{CO}(1-0)}$ of the individual pointings are between $1.2 \pm 0.4$ (position 7) and $<0.6 \pm 0.2$ (position 5). Although these values are within the errors compatible with the above-mentioned reference values (Leroy et al. 2009; Braine et al. 1993), the low value at position 5 could indicate a subthermal excitation and the presence of diffuse gas, which might be responsible for the broad emission feature in the spectrum around $9200 \mathrm{~km} \mathrm{~s}^{-1}$ (Fig. 2).

\subsubsection{Gas-to-dust mass ratio}

The CIGALE fitting of the total IR to submillimeter SED of the disc of UGC 10214 yields a dust mass of $M_{\text {dust }}=(1.7 \pm 0.3) \times$ $10^{8} M_{\odot}$. The total dust-to-gas mass ratio is thus $180 \pm 30$, taking the molecular gas mass from the IRAM measurement. This value is very close to that of the local Galaxy $\left(M_{\text {gas }} / M_{\text {dust }}=186\right.$, taking the He fraction of 1.36 into account, Draine et al. 2007, Table 2), in agreement with the similar metallicity.

\subsubsection{Atomic and molecular gas in VV 29c}

As described in Sect. 2.5, we separated the total HI datacube into the HI emission of VV 29c from that of UGC 10214. We then derived the total spatially integrated HI spectrum from VV $29 \mathrm{c}$ and measured the HI flux by integrating this spectrum over the velocity range between 9630 and $9800 \mathrm{~km} \mathrm{~s}^{-1}$. We obtain a total HI flux of $(0.60 \pm 0.03) \mathrm{Jy} \mathrm{km} \mathrm{s}^{-1}$, and a total HI mass of $(3.5 \pm 0.2) \times 10^{9} M_{\odot}$. This value is very close to that found by Briggs et al. (2001) of $3.1 \times 10^{9} M_{\odot}$ (including helium and for a Hubble constant of $63 \mathrm{~km} \mathrm{~s}^{-1} / \mathrm{Mpc}$ ).

We do not detect any $\mathrm{CO}$ emission from this region and velocity range (see the pv diagram of $\mathrm{CO}$ in Fig. 7). We summed the $\mathrm{CO}$ spectra over the same region where HI emission from VV 29c was detected and determined a $3 \sigma$ upper limit of $0.6 \mathrm{Jy} \mathrm{km} \mathrm{s}^{-1}$, corresponding to a molecular gas mass of $1 \times 10^{8} M_{\odot}$. The molecular gas fraction, $M_{\mathrm{H}_{2}} / M_{\mathrm{HI}}<0.03$, is thus much lower than for UGC 10214, and typical for a dwarf galaxy.

\subsection{Star formation rate}

Both $\mathrm{H} \alpha$ and FUV are SF tracers, although sensitive to different timescales. While $\mathrm{H} \alpha$, produced by ionising stars, traces the current SFR (up to $\sim 10 \mathrm{My}$ ), the FUV emission is emitted mainly by less massive stars with lifetimes of up to $\sim 100 \mathrm{Myr}$. The exact values of these timescales are uncertain and strongly depend on the SF history (see Sect. 5.1 for a discussion). In 


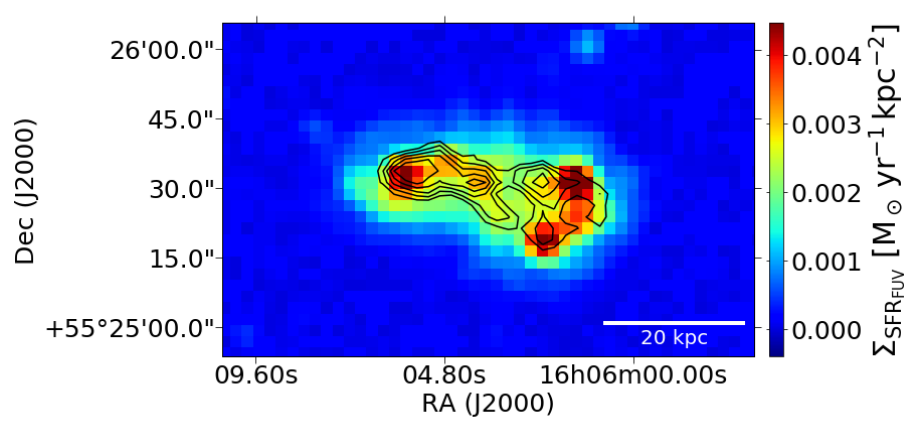

(a)

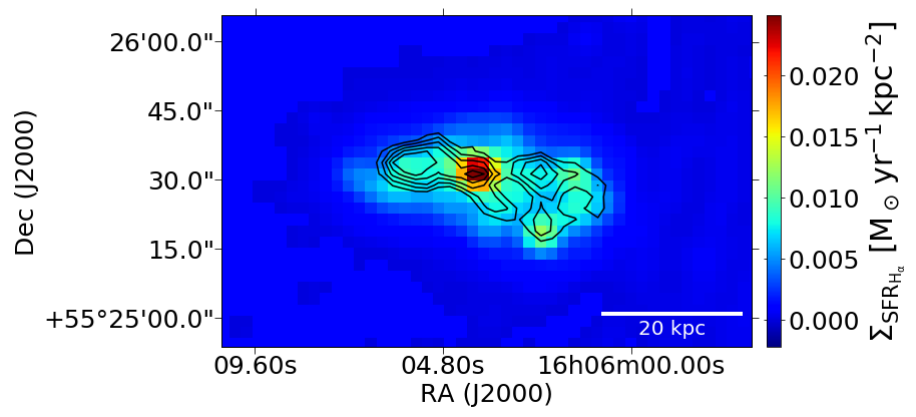

(b)

Fig. 9. CO contour levels (ranging from 6 to $14 \sigma$ in $2 \sigma$ steps) over the maps of panel $a$ : $\mathrm{SFR}_{\mathrm{FUV}}$ and panel $b$ : $\mathrm{SFR}_{\mathrm{H} \alpha}$.

the following we refer to the timescale traced by the FUV as the "recent" SFR, as opposed to the "current" SFR traced by $\mathrm{H} \alpha$. Both tracers are very sensitive to dust extinction. We therefore used hybrid tracers by combining them with the dust re-emission at $24 \mu \mathrm{m}$, in order to also take into account the dust-obscured SF.

We used the prescriptions in Tables 1 and 2 of Kennicutt \& Evans (2012; taken from Kennicutt et al. 2009; Hao et al. 2011; Murphy et al. 2011) to derive SFRs, giving

$\log \mathrm{SFR}_{\mathrm{H}} \alpha\left[M_{\odot} \mathrm{yr}^{-1}\right]=\log (L(\mathrm{H} \alpha)+0.020 L(24 \mu \mathrm{m}))-41.27$,

$\log \mathrm{SFR}_{\mathrm{FUV}}\left[M_{\odot} \mathrm{yr}^{-1}\right]=\log (L(\mathrm{FUV})+3.89 L(24 \mu \mathrm{m}))-43.35$,

where the luminosities are in units of $\operatorname{erg~s}^{-1}$ and $L(24 \mu \mathrm{m})$ and $L(\mathrm{FUV})$ were calculated from the fluxes $F_{\lambda}$ as $L(\lambda)=4 \pi D^{2} \lambda F_{\lambda}$. We assumed following Hao et al. (2011) that IRAS $25 \mu \mathrm{m}$ and MIPS $24 \mu \mathrm{m}$ luminosities can be used interchangeably for this estimation. The SFRs were calculated for a Kroupa initial mass function (IMF; Kroupa 2001), which is very similar to the Chabrier IMF used in the CIGALE modelling.

With these prescriptions, we produced maps for the SFR. Figure 9 shows the resulting maps, overlaid with contours of the CARMA CO image. The different distributions of the SFRs based on $\mathrm{H} \alpha$ and FUV are striking. While $\mathrm{SFR}_{\mathrm{FUV}}$ shows peaks in the east and along the western spiral arm, $\mathrm{SFR}_{\mathrm{H} \alpha}$ has a pronounced maximum in the centre. The ridge towards the east and the western spiral arm are also visible in $\mathrm{SFR}_{\mathrm{H} \alpha}$, but are fainter than the centre. The eastern ridge of $\mathrm{SFR}_{\mathrm{FUV}}$ is closely followed by the $\mathrm{CO}$ emission, which extends until the peak of $\mathrm{SFR}_{\mathrm{H} \alpha}$ in the centre. The western part of the spiral arm is also followed by the $\mathrm{CO}$ and also by $\mathrm{HI}$ (see Fig. 6).

Finally, based on the fluxes from Table 3, we calculated the SFR for each region as well as for the entire disc, and we list them in Table 6. The SFR from the combination of $\mathrm{H} \alpha+24 \mu \mathrm{m}$
$\left(\mathrm{SFR}_{\mathrm{H} \alpha}\right)$ is always higher (by a factor of about 2-4) than the $\mathrm{SFR}$ derived from FUV $+24 \mu \mathrm{m}\left(\mathrm{SFR}_{\mathrm{FUV}}\right)$. The difference is more pronounced in the centre (region 2). This trend is also seen in the modelling results with CIGALE (Table 5), where the values for the current SFR (SFR 10 Myr which corresponds to the timescale of $\mathrm{H} \alpha$ ) are considerably higher (factor 3.5-6) than for the SFR in the past $100 \mathrm{Myr}, \mathrm{SFR}_{100 \mathrm{Myr}}$. The values for $\mathrm{SFR}_{\mathrm{H} \alpha}$ and $S_{10 M y r}$ generally agree very well (expect for region 1), but the values for $S_{F R}$ FU are a factor of 1.5-2 higher than $\mathrm{SFR}_{100 \mathrm{Myr}}$. A reason for this discrepancy might be that the timescale for the FUV-traced SFR is of the order of several tens to $100 \mathrm{Myr}$ (see Sect. 5.1), so that SFR $_{\mathrm{FUV}}$ traces a somewhat shorter time than $\mathrm{SFR}_{100 \mathrm{Myr}}$. In the case of a decreasing SFR, as modelled here, the SFR averaged over a shorter timescale results in a higher value. In any case, both methods for deriving the SF history indicate that the SFR has increased in the recent past.

In Table 6 we also list the molecular gas mass (from Eq. (1)) for the different regions and the resulting molecular gas depletion time, $\tau_{\text {dep }}=M_{\mathrm{H}_{2}} / \mathrm{SFR}$. The current molecular gas depletion time (from $\mathrm{SFR}_{\mathrm{H} \alpha}$ ) is very close to the value found by Bigiel et al. (2011), and the molecular gas depletion time from $\mathrm{SFR}_{\mathrm{FUV}}$ is a factor $\sim 2$ lower. The variations of $\tau_{\text {dep }}$ between the different regions are less than a factor 2 , with a trend of a shorter $\tau_{\text {dep }}$ in the western side (region 3).

\subsection{Stellar mass}

The IRAC $3.6 \mu \mathrm{m}$ emission is a good tracer of the low-mass stars that dominate the total stellar mass. We therefore used the $3.6 \mu \mathrm{m}$ emission as a tracer for the stellar mass, following as a starting point the calibration by Oliver et al. (2010) for the morphological type of UGC $10214(\mathrm{Sb}(\mathrm{s}) \mathrm{c}$, according to the NASA Extragalactic Database), $M_{\star}\left[M_{\odot}\right]=27.6 L(3.6 \mu \mathrm{m})\left[L_{\odot}\right]$. This calibration is based on a Salpeter IMF, and we applied a correction factor of 0.626 to convert this expression into a Chabrier IMF and to achieve agreement with the stellar mass from CIGALE for the entire disc. Thus, we calculated the stellar mass as

$\frac{M_{\star}}{M_{\odot}}=14.5 \times \frac{L(3.6 \mu \mathrm{m})}{L_{\odot}}$.

Based on the fluxes from Table 3, we calculated the stellar mass for each region as well as for the entire disc (Table 6). These values for $M_{\star}$ for region 1-3 agree within the errors with those calculated with CIGALE (Table 5), showing that the simple prescription of Eq. (9) is a good approximation. In the central region of the galaxy (region 2), the stellar mass is higher and the specific SFR, $\mathrm{sSFR}=\mathrm{SFR} / M_{\star}$, is lower, in particular when considering $\mathrm{SFR}_{\mathrm{FUV}}$. The highest values of sSFR are in the western part for $\mathrm{SFR}_{\mathrm{FUV}}$ and for $\mathrm{SFR}_{\mathrm{H} \alpha}$.

\subsection{Pixel-by-pixel analysis of star formation and gas}

\subsubsection{Kennicutt-Schmidt law}

Figure 10 shows the SFR per area as a function of the surface density of the molecular gas (the Kennicutt-Schmidt relation) for the individual pixels in the images. We show the relation for both $\mathrm{SFR}_{\mathrm{H} \alpha}$ and $\mathrm{SFR}_{\mathrm{FUV}}$ for regions 1, 2, and 3 separately. We furthermore indicate the distance from the centre as the colour coding.

The most striking result is that $\Sigma_{\mathrm{SFR}}$ is systematically higher than $\Sigma_{\mathrm{SFR}}$ for a given molecular mass surface density. 
Table 6. Values of SFR, $M_{\star}, M_{\mathrm{H}_{2}}$, sSFR, and molecular gas depletion time estimated for the disc and the three disc regions.

\begin{tabular}{|c|c|c|c|c|}
\hline Band & Total & Reg 1 & $\operatorname{Reg} 2$ & $\operatorname{Reg} 3$ \\
\hline $\mathrm{SFR}_{\mathrm{FUV}}\left[M_{\odot} \mathrm{yr}^{-1}\right]$ & $1.66 \pm 0.17$ & $0.44 \pm 0.04$ & $0.47 \pm 0.05$ & $0.61 \pm 0.06$ \\
\hline $\mathrm{SFR}_{\mathrm{H} \alpha}\left[M_{\odot} \mathrm{yr}^{-1}\right]$ & $4.73 \pm 0.19$ & $1.11 \pm 0.05$ & $1.90 \pm 0.07$ & $1.49 \pm 0.06$ \\
\hline$M_{\star}\left[10^{9} M_{\odot}\right]$ & $128 \pm 13$ & $32 \pm 3$ & $64 \pm 6$ & $28 \pm 3$ \\
\hline$M_{\mathrm{H}_{2}}\left[10^{9} M_{\odot}\right](\mathrm{CARMA})$ & $6.0 \pm 0.6$ & $1.9 \pm 0.2$ & $2.4 \pm 0.2$ & $1.7 \pm 0.2$ \\
\hline$M_{\mathrm{H}_{2}}\left[10^{9} M_{\odot}\right]($ IRAM $)$ & $9.0 \pm 1.5$ & & & \\
\hline$M_{\mathrm{HI}}\left[10^{9} M_{\odot}\right]$ & $22.1 \pm 0.7$ & - & - & - \\
\hline sSFR (FUV) $\left[10^{-12} \mathrm{yr}^{-1}\right]$ & $13 \pm 3$ & $14 \pm 3$ & $7.3 \pm 1.5$ & $22 \pm 4$ \\
\hline $\operatorname{sSFR}(\mathrm{H} \alpha)\left[10^{-12} \mathrm{yr}^{-1}\right]$ & $36 \pm 5$ & $35 \pm 5$ & $30 \pm 4$ & $53 \pm 8$ \\
\hline$\tau_{\text {dep }}{ }^{a}$ (FUV) [Gyr] (CARMA) & $3.6 \pm 0.7$ & $4.4 \pm 0.9$ & $5.1 \pm 1.0$ & $2.8 \pm 0.6$ \\
\hline$\tau_{\text {dep }}{ }^{a}(\mathrm{FUV})[\mathrm{Gyr}](\mathrm{IRAM})$ & $5.4 \pm 1.4$ & & & \\
\hline$\tau_{\mathrm{dep}}{ }^{a}(\mathrm{H} \alpha)[\mathrm{Gyr}](\mathrm{CARMA})$ & $1.3 \pm 0.2$ & $1.75 \pm 0.25$ & $1.3 \pm 0.2$ & $1.1 \pm 0.2$ \\
\hline$\tau_{\mathrm{dep}}{ }^{a}(\mathrm{H} \alpha)[\mathrm{Gyr}](\mathrm{IRAM})$ & $1.9 \pm 0.4$ & & & \\
\hline
\end{tabular}

Notes. ${ }^{(a)}$ Molecular gas depletion time $\tau_{\text {dep }}=\mathrm{SFR} / M_{\mathrm{H}_{2}}$.

This effect has already been seen in the global and regional analysis (Sect. 4.2) and indicates a higher current SFR, traced by $\mathrm{H} \alpha$, than the recent SFR, traced by FUV. The pixel-to-pixel analysis shows that this trend is present over the entire disc, and that it is particularly pronounced in region 2 (the central region), where the molecular depletion time, $\tau_{\text {dep }}$, is about $0.8-0.9$ dex lower for $\mathrm{SFR}_{\mathrm{H} \alpha}$ than for $\mathrm{SFR}_{\mathrm{FUV}}$. The difference in the other regions is less pronounced $(\sim 0.4 \mathrm{dex})$.

In regions 1 and 3 , the relation between $\Sigma_{\mathrm{SFR}}$ and $\Sigma_{\text {mol }}$ has similar shapes for the FUV- and $\mathrm{H} \alpha$-traced SFRs, the only difference being the systematically higher values for $\Sigma_{\mathrm{SFR}_{\mathrm{H} \alpha}}$. This means that the increase in the current SFR has taken place over the entire galaxy. The mean molecular gas depletion times are slightly shorter (by $0.2 \mathrm{dex}$ ) in region 3 than in region 1 for $\mathrm{SFR}_{\mathrm{H} \alpha}$ and for $\mathrm{SFR}_{\mathrm{FUV}}$. In region 2 , the situation is different. While $\Sigma_{\mathrm{SFR}_{\mathrm{H} \alpha}}$ shows a roughly linear increase with $\Sigma_{\mathrm{mol}}$, the relation of $\Sigma_{\mathrm{SFR} F u}$ with $\Sigma_{\text {mol }}$ is flat, which means that the molecular depletion time, $\tau_{\text {dep, FUv }}$, changes systematically as a function of $\Sigma_{\text {mol }}$ and as a function of distance to the centre, while at large central distance, $\tau_{\text {dep,FUV }}$ is similar to the values in regions 1 and 3 , closer to the centre $\tau_{\text {dep,FUV }} \approx 10 \mathrm{Gyr}$.

The mean values for the molecular depletion times, averaged over all pixels, are $\tau_{\text {dep, FUV }}=6.9 \mathrm{Gyr}$ (with a standard deviation of $2.5 \mathrm{Gyr}$ ) and $\tau_{\mathrm{dep}, \mathrm{H} \alpha}=2.4 \mathrm{Gyr}$ (with a standard deviation of $1.0 \mathrm{Gyr}$ ). The depletion time from $\mathrm{H} \alpha$ is very similiar to the result of Bigiel et al. (2011) from a spatially resolved analysis of 30 spiral galaxies based on FUV $+24 \mu \mathrm{m}$ as an SFR tracer $\left(\tau_{\text {dep }}\right.$ $=2.35 \mathrm{Gyr}$ with a dispersion of $0.24 \mathrm{dex}$ ), while $\tau_{\text {dep, FUV }}$ is a factor of 2.9 longer $\left(0.46\right.$ dex below). The long $\tau_{\text {dep, FUV may }}$ reflect the changes in the SF history rather than indicating a low star formation efficiency (SFE, the inverse of the gas depletion time) because the molecular gas that we observe is related to the current SFR (traced by $\mathrm{H} \alpha$ ) and not to the SF several tens of Myr ago.

\subsubsection{SFR and stellar mass}

Figure 11 shows the surface density of $\mathrm{SFR}_{\mathrm{FUV}}$ and $\mathrm{SFR}_{\mathrm{H} \alpha}$ as a function of stellar mass surface density for the three regions, colour-coded with the radial distance from the centre. Overlaid as a pink ellipsoid is the spatially resolved (on kpc scale) MS found by Ellison et al. (2018) for a sample of 1390 star-forming galaxies from the Sloan Digital Sky Survey Mapping Galaxies at Apache Point Observatory (MaNGA) survey. Similar to the results from the previous section, we find that (i) $\Sigma_{\mathrm{SFR}_{\mathrm{FUV}}}$ is systematically lower than $\Sigma_{\mathrm{SFR}}$ for a given $\Sigma_{M_{\star}}$, (ii) the relation between $\Sigma_{\mathrm{SFR}}$ and $\Sigma_{M_{\star}}$ has a similar shape for the FUV- and $\mathrm{H} \alpha$ traced SFRs in regions 1 and 3, but not in region 2, where the FUV relation is much flatter, and (iii) the ratio $\Sigma_{\mathrm{SFR}_{\mathrm{FUV}}} / \Sigma_{\mathrm{SFR}}$ is particularly low in the central region.

In addition to these results, we can draw further conclusions from a more detailed comparison of $\Sigma_{\text {SFR }}$ with $\Sigma_{M_{\star}}$ and in particular with the MS found for SF galaxies. The $\mathrm{H} \alpha$-traced relation falls almost entirely within the range of the MS found by Ellison et al. (2018). Only the most central regions lie outside, but they fall outside the stellar surface density range covered by the Ellison et al. (2018) sample so that a direct comparison is not possible. The situation is different for the FUV-traced relation. In region 2 almost all pixels, except for those at the largest radial distances, have $\Sigma_{\mathrm{SFR}}$ below the MS value for the corresponding $\Sigma_{M_{\star}}$. In region 1 , the innermost pixels are also slightly below the MS. In region 3, all pixels lie within the MS. Here, the sSFR $\left(\Sigma_{\mathrm{SFR}} / \Sigma_{M_{\star}}\right)$ is about a factor 2 higher than in region 1.

Taken together, these results indicate that UGC 10214 is currently forming stars at a rate corresponding to MS galaxies in practically the entire galaxy. For the recent SFR, traced by FUV, the picture is more heterogenous. While the SF in region 3 is entirely within the MS and in region 1 is slightly below the MS for the innermost points, it is well below the MS in region 2, indicating a supression of the SF especially in the central part.

\section{Discussion}

\subsection{Timescale of the SF tracers}

Our dataset and analysis have revealed very noticeable differences between the values and the distribution of the $\mathrm{H} \alpha$ - and FUV-traced SFRs that are likely due to temporal changes in the SFR. The exact timescales over which each tracer probes the SFR are difficult to quantify. They depend on the definition of this timescale and on the SF history. Boquien et al. (2014) investigated this issue for different SF histories and found that the $\mathrm{H} \alpha$ emission, produced by ionising stars, has a luminosity-weighted age of those stars that are contributing to $\mathrm{H} \alpha$ of 2-4 Myr (for a constant SFR during the past $100 \mathrm{Myr}$ or $1 \mathrm{Gyr}$ ). The luminosityweighted age of the FUV emission is older, $\sim 15 \mathrm{Myr}$, for a constant SFR during the past $100 \mathrm{Myr}$, and 50 Myr for a constant SFR during the past Gyr. Adopting as the timescale the moment 

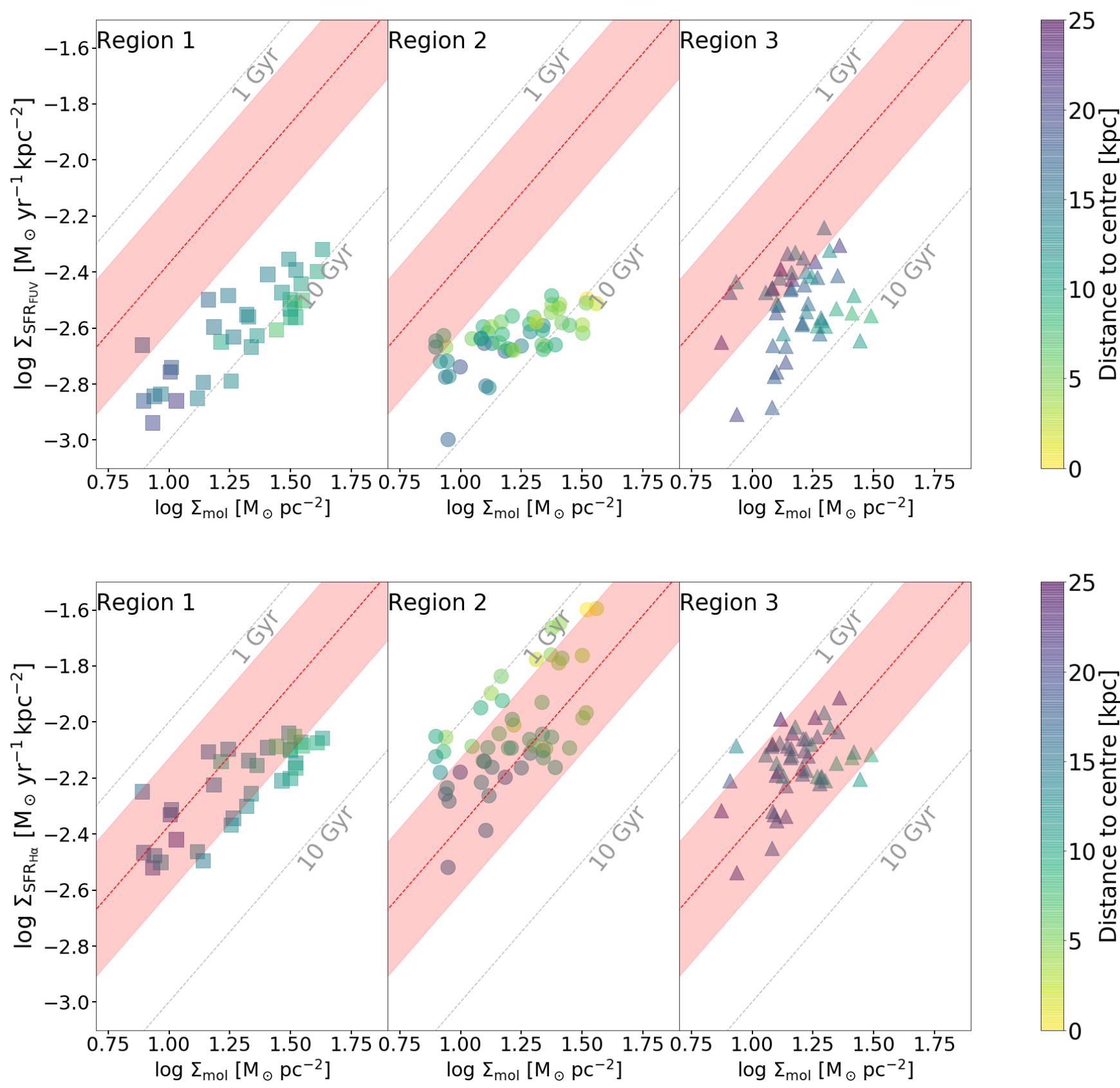

Fig. 10. SFR per area vs. molecular gas surface density (Kennicutt-Schmidt relation) for different regions in the disc of UGC 10214. Upper row: SFR derived from FUV and $24 \mu \mathrm{m}$ emission, $\mathrm{SFR}_{\mathrm{FUV}}$, and lower row: SFR derived from the $\mathrm{H} \alpha$ and $24 \mu \mathrm{m}$ emissions, $\mathrm{SFR}_{\mathrm{H} \alpha}$. The pixels in the separate panels correspond to the different regions shown in Fig. 1. The dashed grey lines represent different molecular gas depletion times. The dashed red line represents the depletion time estimated for star-forming galaxies (2.35 Gyr, Bigiel et al. 2011) and its dispersion (0.23 dex), represented by the pink-shaded area. The colour of the markers of the different regions indicates the distance to the galaxy centre.

when an instantaneous burst is down to $10 \%$ of its initial luminosity, we obtain a timescale for the FUV of $100 \mathrm{Myr}$ and for the $\mathrm{H} \alpha$ emission of $10 \mathrm{Myr}$ (Kennicutt \& Evans 2012, their Table 2). Thus, while the timescale for the $\mathrm{H} \alpha$ emission is always $\leq 10 \mathrm{Myr}$, the timescale for the FUV depends on its definition and on the SF history and can take values between several tens of Myr to 100 Myr.

We used hybrid SF tracers by including the $24 \mu \mathrm{m}$ emission, thus the $24 \mu \mathrm{m}$ timescale has also to be taken into account. Boquien et al. (2014) derived luminosity-averaged ages of the total infrared (TIR) luminosity of $15 \mathrm{Myr}$ (for a constant SFR over the past $100 \mathrm{Myr}$ ) and of $100 \mathrm{Myr}$ (for a constant SFR over the last Gyr), which is similar to the timescale of the FUV. The $24 \mu \mathrm{m}$ emission, emitted mostly from very small dust grains heated by massive stars, might have a shorter timescale. Thus, the timescale of the hybrid $\mathrm{H} \alpha+24 \mu \mathrm{m}$ emission might be longer than that of $\mathrm{H} \alpha$ alone. However, the $24 \mu \mathrm{m}$ emission contributes only $20 \%$ or less (depending on the region) to $\mathrm{SFR}_{\mathrm{H} \alpha}$, so that we do not expect it to have a large effect on the timescale. The timescales of the FUV and $24 \mu \mathrm{m}$ are (within the uncertainties) similar.

We therefore conclude that the $\mathrm{H} \alpha+24 \mu \mathrm{m}$ emission traces the current SFR on a timescale of $\lesssim 10 \mathrm{Myr}$, while FUV $+24 \mu \mathrm{m}$ traces the recent SFR on a timescale of several tens of Myr. 

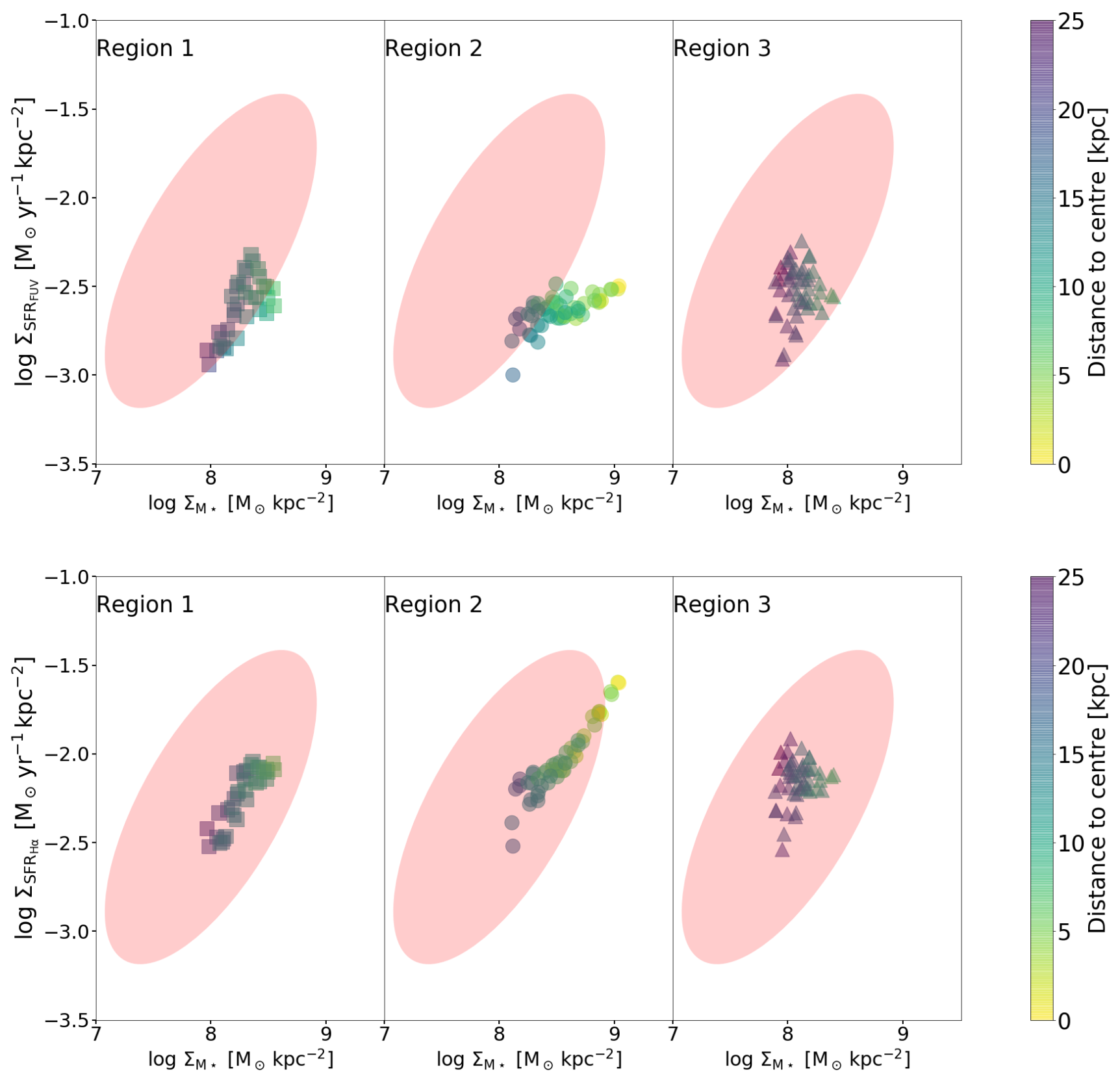

Fig. 11. SFR per area vs. stellar mass surface density for different regions in the disc of UGC 10214. Upper row: SFR derived from the FUV and $24 \mu \mathrm{m}$ emissions, $\mathrm{SFR}_{\mathrm{FUV}}$, and lower row: SFR derived from the $\mathrm{H} \alpha$ and $24 \mu \mathrm{m}$ emission, $\mathrm{SFR}_{\mathrm{H} \alpha}$. The pixels in the separate panels correspond to the different regions shown in Fig. 1. The colour of the markers of the different regions indicates the distance to the galaxy centre. For comparison, the pink ellipse shows the location of the resolved MS from Ellison et al. (2018).

\subsection{Effect of the minor interaction on UGC 10214}

The interaction with the dwarf galaxy VV 29c has had a very noticeable effect on the morphology of UGC 10214. A long tidal tail has formed, containing a large portion of the atomic gas ( $\sim 40 \%)$ as well as bright young stellar clusters. In addition, the morphology of the stellar disc is disturbed and the gas distribution, both atomic and molecular, is asymmetric.

Our dataset and analysis have revealed very noticeable changes of the distribution and rate of the SF in the past tens of Myr. After a period of suppressed SF in the central region several tens of Myr ago, the SFR has increased globally and changed its location from the outer spiral arms where bright knots are visible in the FUV and $24 \mu \mathrm{m}$ emission to the centre where the most active $\mathrm{SF}$ is currently taking place.
Have these changes of the SF rate and distribution been caused by the minor interaction? Following Tran et al. (2003), we can estimate when the tail-forming event took place by dividing the length of the tidal tail $(\sim 100 \mathrm{kpc})$ by the expected gas ejection velocity, which we adopt as similar to the galaxy rotation velocity (about $400 \mathrm{~km} \mathrm{~s}^{-1}$ from the $\mathrm{HI}$ ), yielding 250 Myr. Thus, from a timescale point of view, the minor interaction might very well have caused the changes in the SF activity. The presence of star clusters, some of which are very bright (see the supercluster found and analysed by Tran et al. 2003) in the disc and tail, which are younger than this dynamical timescale, also suggests that their formation has possibly been triggered by the event. The minor merger is very likely responsible for the unusual distribution of the SF in the disc, with bright SF knots, visible in $8 \mu \mathrm{m}, 24 \mu \mathrm{m}$, and FUV in the spiral arms. The ring-like 
Table 7. Optical and IR colours and mass ratios of UGC 10214.

\begin{tabular}{ll}
\hline \hline$u_{\text {corr }}^{\prime} a$ & $2.73 \mathrm{mJy}$ \\
$r_{\text {corr }}^{\prime}$ & $18.43 \mathrm{mJy}$ \\
$\left(u^{\prime}-r^{\prime}\right)_{\text {corr }}$ & $2.1 \mathrm{mag}$ \\
\hline$M_{\mathrm{H}_{2}} / M_{\mathrm{HI}}$ & $0.40 \pm 0.07$ \\
$M_{\mathrm{H}_{2}} / M_{\star}$ & $0.07 \pm 0.01$ \\
$M_{\text {gas }} / M_{\star}$ & $0.24 \pm 0.02(0.34 \pm 0.02)^{b}$ \\
\hline
\end{tabular}

Notes. ${ }^{(a)}$ Corrected for Galactic extinction and internal extinction from CIGALE. ${ }^{(b)}$ Including the atomic masses of the disc and the tails $(1.2 \times$ $10^{10} M_{\odot}$ ) (Knierman et al., in prep.).

structure in the western arm might indicate, as suggested by Jarrett et al. (2006), that the interaction was an off-centre collisional interaction. The current (moderate) increase in the SF in the centre, visible in $\mathrm{H} \alpha$, might have been caused by a gas flow towards the centre. The thin CO filament connecting the eastern side to the nucleus, visible in the CARMA map, is a hint for this, together with the broad IRAM CO spectra (widths of $500 \mathrm{~km} \mathrm{~s}^{-1}$ ) at the corresponding positions 2 and 3 .

The gas properties, on the other hand, do not seem to be considerably affected by the past interaction. The pv diagram shows a regular rotation curve with no indication of a major perturbation, even though at the higher velocity resolution of the IRAM spectra, the line shapes are unusual at some positions. The fact that the average $\mathrm{H} \alpha$-based molecular gas depletion time is normal fits into this picture and shows a galaxy in which the star formation seems to proceed in the same way as in undisturbed spiral galaxies.

\subsection{Evolutionary phase of UGC 10214}

UGC 10214 is a massive galaxy, close to the upper end of the mass distribution of galaxies. Our multi-wavelength dataset allows us to determine its current evolutionary stage and predict its future evolution.

In order to determine the location of UGC 10214 on the galaxy MS, we compared its SFR and stellar mass to a sample consisting of 12006 SDSS galaxies with $M_{\star}>10^{10} M_{\odot}$ used in Saintonge et al. (2016) as the parent distribution for their COLDGASS study. This mass range is adequate for our object, which is important because the relation between SFR and $M_{\star}$ flattens considerably above galaxy masses of about $10^{10} M_{\odot}$, (Noeske et al. 2007; Schreiber et al. 2016), that is, the SFR per stellar mass decreases for high stellar masses. The reason for this flattening is not clear. We find from a comparison to Fig. 1 and Eq. (5) of Saintonge et al. (2016) that UGC 10214 falls into the range of MS SF galaxies, with SFR $_{\mathrm{FUV}}$ lying right on the MS and $\mathrm{SFR}_{\mathrm{H} \alpha}$ a factor 2.5 above. Thus, in spite of the minor merger, UGC 10214 has an SFR that is normal for its mass.

The extinction-corrected optical colour $u^{\prime}-r^{\prime}$ (see Table. 7), corrected for internal extinction, locates UGC 10214 in the middle of the green valley (Alatalo et al. 2014; Schiminovich et al. 2007). This apparent contradiction (lying on the star-forming MS but at the same time in the optical green valley) has its explanation most likely in the high stellar mass of UGC 10214 and is not unusual. Cortese (2012) found that red spirals, selected based on their optical colours, were not quiescent but formed stars at a normal $\left(>1 M_{\odot} \mathrm{yr}^{-1}\right)$ rate and showed UV-optical colours typical for the blue cloud. The authors concluded that at high stellar masses, optical colours do not allow distinguishing between actively SF and quiescent objects. This is also the case for UGC 10214, which has relatively red optical colours due to the bulk of stars formed in earlier times, which dominate the optical colours independent of the current SFR. The low sSFR (see Table 6) reflects this as well. Additionally, the minor merger that has most likely produced an increase in the SFR in the past might have moved UGC 10214 from below right on the star-forming MS.

Thus, UGC 10214 is a massive spiral galaxy with a normal SFR and molecular depletion time. As discussed in the previous section, the minor merger has most likely caused a moderate increase of the SFR but has not perturbed the gas significantly. The total gas fraction $\left(M_{\mathrm{gas}} / M_{\star}\right)$ and molecular gas fraction $\left(M_{\mathrm{H}_{2}} / M_{\mathrm{HI}}\right)$ are normal for a galaxy of its mass (see Saintonge et al. 2016). The gas reservoir is enough to continue SF for another 5-10 Gyr with increasing stellar mass significantly in the future.

\section{Summary and conclusions}

We presented a multi-wavelength analysis of the disc galaxy UGC 10214, which has experienced a minor interaction with the dwarf galaxy VV 29c about $250 \mathrm{Myr}$ ago. These data allowed us to measure the total atomic gas mass, dust mass, molecular gas mass and surface density, the SFRs on different timescales (based on the $\mathrm{H} \alpha$ and FUV emissions), and the stellar mass. In addition, we carried out SED fittings with CIGALE for the entire disc and for three different regions. Our results can be summarised as follows.

- UGC 10214 is a massive galaxy, $M_{\star}=(1.28 \pm 0.13) \times$ $10^{11} M_{\odot}$, with a moderate current $\mathrm{SFR}, \mathrm{SFR}_{\mathrm{H} \alpha}=$ $(4.67 \pm 0.19) M_{\odot} \mathrm{yr}^{-1}$.

- We measured the total atomic, $(2.21 \pm 0.07) \times 10^{10} M_{\odot}$, and molecular, $(9.0 \pm 1.5) \times 10^{9} M_{\odot}$, gas mass of UGC 10214. The molecular gas fraction, $M_{\mathrm{H}_{2}} / M_{\mathrm{HI}}=0.40 \pm 0.07$, shows that UGC 10214 is a molecular gas-rich galaxy. The total gas fraction, $M_{\text {gas }} / M_{\star}=0.2 \pm 0.03$, and the gas-to-dust mass ratio is 180 (including $\mathrm{He}$ ), similar to the value of the Milky Way.

- We measured the atomic gas mass of the companion dwarf galaxy VV 29c, $(3.5 \pm 0.2) \times 10^{9} M_{\odot}$. We did not detect any $\mathrm{CO}$ emission at the position and velocity of VV 29c with an upper limit of the molecular gas mass as $10^{8} M_{\odot}$. The molecular gas fraction in this object is thus low $(<0.03)$, which is typical for a dwarf galaxy.

- The molecular and atomic gas have an irregular distribution throughout the disc of UGC 10214. The general gas kinematics, on the other hand, shows a regular rotation pattern with no visible indications of major perturbations.

- The comparison of the SFR derived from the FUV, SFR $\mathrm{FUV}_{\text {, }}$ and from the $\mathrm{H} \alpha$ emission, $\mathrm{SFR}_{\mathrm{H} \alpha}$, reveals that the distribution of the SF has changed drastically in the recent past. While SFR $_{\text {FUV }}$ is concentrated in the outer spirals arms, $\mathrm{SFR}_{\mathrm{H} \alpha}$ shows a pronounced peak in the centre. This shows that there has been a shift of the SFR from the outer disc to the centre in the past 10-100 Myr.

- The SFR has increased globally by a factor of 2-3 in the recent past. This increase has been particularly strong in the centre (factor of $\sim 4$ ), where a peak in the current star formation is found. While the current SFR lies everywhere within the spatially resolved MS, the recent FUV-derived SFR in the central regions is below the MS.

- The current molecular gas depletion time, $\tau_{\mathrm{dep}, \mathrm{H} \alpha}$, averaged over the entire disc, is $(1.9 \pm 0.4) \mathrm{Gyr}$, similar to the mean value found for spiral galaxies.

- The optical shows that UGC 10214 is in the optical green valley, but its SFR follows the galaxy SF main sequence. The 
most likely reason for this apparent discrepancy is the high stellar mass that dominates the optical colours.

These results show that UGC 10214 is a massive spiral galaxy with a normal (with respect to its stellar mass) SFR and a normal molecular gas depletion time. The minor merger has most likely caused variations in the SFR during the past $\sim \mathrm{Myr}$ and a moderate increase in the SFR. It does not seem to have perturbed the gas significantly at the present time.

Acknowledgements. DRB acknowledges financial support from the Spanish Ministry of Economy and Competitiveness (MINECO) under the grant number AYA2016-76219-P. UL and SV acknowledge support by the research projects AYA2014-53506-P and AYA2017-84897-P from the Spanish Ministerio de Economía y Competitividad, from the European Regional Development Funds (FEDER) and the Junta de Andalucía (Spain) grants FQM108. This study has been partially financed by the Consejería de Conocimiento, Investigación y Universidad, Junta de Andalucía and European Regional Development Fund (ERDF), ref. SOMM17/6105/UGR. KK is supported by an NSF Astron omy and Astrophysics Postdoctoral Fellowship under award AST-1501294. MB acknowledges support from FONDECYT regular grant 1170618. We acknowl edge the usage of the HyperLeda database (http://leda.univ-lyon 1 . fr and of the Nasa Extragalactic Database (NED; https://ned.ipac.caltech edu). Based on observations made with the NASA Galaxy Evolution Explorer. GALEX is operated for NASA by the California Institute of Technology under NASA contract NAS5-98034. Funding for SDSS-III has been provided by the Alfred P. Sloan Foundation, the Participating Institutions, the National Science Foundation, and the U.S. Department of Energy Office of Science. The SDSS III web site is http://www.sdss3.org/. SDSS-III is managed by the Astrophysical Research Consortium for the Participating Institutions of the SDSS-III Collaboration including the University of Arizona, the Brazilian Participation Group, Brookhaven National Laboratory, University of Cambridge, Carnegie Mellon University, University of Florida, the French Participation Group, the German Participation Group, Harvard University, the Instituto de Astrofisica de Canarias, the Michigan State/Notre Dame/JINA Participation Group, Johns Hopkins University, Lawrence Berkeley National Laboratory, Max Planck Institute for Astrophysics, Max Planck Institute for Extraterrestrial Physics, New Mexico State University, New York University, Ohio State University, Pennsylvania State University, University of Portsmouth, Princeton University, the Spanish Participation Group, University of Tokyo, University of Utah, Vanderbilt University, University of Virginia, University of Washington, and Yale University. The Spitzer Space Telescope is operated by the Jet Propulsion Laboratory, California Institute of Technology, under contract with the National Aeronautics and Space Administration. SPIRE has been developed by a consortium of institutes led by Cardiff University (UK) and including Univ. Lethbridge (Canada); NAOC (China); CEA, LAM (France); IFSI, Univ Padua (Italy); IAC (Spain); Stockholm Observatory (Sweden); Imperial College London, RAL, UCL-MSSL, UKATC, Univ. Sussex (UK); and Caltech, JPL, NHSC, Univ. colourado (USA). This development has been supported by national funding agencies: CSA (Canada); NAOC (China); CEA, CNES CNRS (France); ASI (Italy); MCINN (Spain); SNSB (Sweden); STFC, UKSA (UK); and NASA (USA). This work is based on observations carried out unde project number 062-05 with the IRAM 30m telescope. IRAM is supported by INSU/CNRS (France), MPG (Germany) and IGN (Spain). The National Radio Astronomy Observatory is a facility of the National Science Foundation operated under cooperative agreement by Associated Universities, Inc. IRAF is distributed by the National Optical Astronomy Observatory, which is operated by the Association of Universities for Research in Astronomy (AURA) under cooperative agreement with the National Science Foundation (Tody 1993) This research made use of Astropy, a community-developed core PythoN (http://www.python.org) package for Astronomy (Astropy Collaboration 2013, 2018); IPYTHON (Pérez \& Granger 2007); MATPLOtLiB (Hunter 2007); and NumPy (van der Walt et al. 2011).

\section{References}

Alatalo, K., Cales, S. L., Appleton, P. N., et al. 2014, ApJ, 794, L13 Aniano, G., Draine, B. T., Gordon, K. D., \& Sandstrom, K. 2011, PASP, 123 1218

Argudo-Fernández, M., Verley, S., Bergond, G., et al. 2015, A\&A, 578, A110

Argudo-Fernández, M., Shen, S., Sabater, J., et al. 2016, A\&A, 592, A30

Astropy Collaboration (Robitaille, T. P., et al.) 2013, A\&A, 558, A33

Astropy Collaboration (Price-Whelan, A. M., et al.) 2018, AJ, 156, 123

Bell, E. F., Phleps, S., Somerville, R. S., et al. 2006, ApJ, 652, 270
Bigiel, F., Leroy, A. K., Walter, F., et al. 2011, ApJ, 730, L13 Bolatto, A. D., Wolfire, M., \& Leroy, A. K. 2013, ARA\&A, 51, 207 Boquien, M., Buat, V., \& Perret, V. 2014, A\&A, 571, A72 Boquien, M., Burgarella, D., Roehlly, Y., et al. 2019, A\&A, 622, A103 Bournaud, F., \& Duc, P.-A. 2006, A\&A, 456, 481

Bournaud, F., Jog, C. J., \& Combes, F. 2005, A\&A, 437, 69 Bradley, L., Sipocz, B., \& Robitaille, T. 2017, astropy/photutils: v0.4 Braine, J., Combes, F., Casoli, F., et al. 1993, A\&AS, 97, 887

Briggs, D. S. 1995, PhD Thesis, New Mexico Institute of Mining Technology, Socorro, New Mexico, USA

Briggs, F. H., Möller, O., Higdon, J. L., Trentham, N., \& Ramirez-Ruiz, E. 2001, A\&A, 380, 418

Brinchmann, J., Charlot, S., White, S. D. M., et al. 2004, MNRAS, 351, 1151

Bruzual, G., \& Charlot, S. 2003, MNRAS, 344, 1000

Calzetti, D., Armus, L., Bohlin, R. C., et al. 2000, ApJ, 533, 682

Cardelli, J. A., Clayton, G. C., \& Mathis, J. S. 1989, ApJ, 345, 245

Cortese, L. 2012, A\&A, 543, A132

Dale, D. A., Helou, G., Magdis, G. E., et al. 2014, ApJ, 784, 83

de Grijs, R., Lee, J. T., Clemencia Mora Herrera, M., et al. 2003, New A, 8 , 155

Draine, B. T., Dale, D. A., Bendo, G., et al. 2007, ApJ, 663, 866

Draine, B. T., Aniano, G., Krause, O., et al. 2014, ApJ, 780, 172

Dressler, A. 1980, ApJ, 236, 351

Duplancic, F., Alonso, S., Lambas, D. G., \& O’Mill, A. L. 2015, MNRAS, 447 1399

Ellison, S. L., Patton, D. R., Simard, L., et al. 2010, MNRAS, 407, 1514

Ellison, S. L., Teimoorinia, H., Rosario, D. J., \& Mendel, J. T. 2016, MNRAS, 458, L34

Ellison, S. L., Sánchez, S. F., Ibarra-Medel, H., et al. 2018, MNRAS, 474, 2039

Fazio, G. G., Hora, J. L., Allen, L. E., et al. 2004, ApJS, 154, 10

Ferland, G. J., Korista, K. T., Verner, D. A., et al. 1998, PASP, 110, 761

Griffin, M. J., Abergel, A., Abreu, A., et al. 2010, A\&A, 518, L3

Hao, C.-N., Kennicutt, R. C., Johnson, B. D., et al. 2011, ApJ, 741, 124

Hickson, P., de Mens Oliveira, C., Huchra, J. P., \& Palumbo, G. G. 1992, ApJ, 399,353

Hunter, J. D. 2007, Comput. Sci. Eng., 9, 90

Inoue, A. K. 2011, MNRAS, 415, 2920

Jarrett, T. H., Polletta, M., Fournon, I. P., et al. 2006, AJ, 131, 261

Kaviraj, S., Darg, D., Lintott, C., Schawinski, K., \& Silk, J. 2012, MNRAS, 419, 70

Kennicutt, R. C., \& Evans, N. J. 2012, ARA\&A, 50, 531

Kennicutt, Jr., R. C., Hao, C.-N., Calzetti, D., et al. 2009, ApJ, 703, 1672

Kroupa, P. 2001, MNRAS, 322, 231

Leroy, A. K., Walter, F., Bigiel, F., et al. 2009, AJ, 137, 4670

Lisenfeld, U., Alatalo, K., Zucker, C., et al. 2017, A\&A, 607, A110

Martin, D. C., Fanson, J., Schiminovich, D., et al. 2005, ApJ, 619, L1

Moore, B., Katz, N., Lake, G., Dressler, A., \& Oemler, A. 1996, Nature, 379, 613

Morrissey, P., Conrow, T., Barlow, T. A., et al. 2007, ApJS, 173, 682

Mould, J. R., Huchra, J. P., Freedman, W. L., et al. 2000, ApJ, 529, 786

Murphy, E. J., Condon, J. J., Schinnerer, E., et al. 2011, ApJ, 737, 67

Noeske, K. G., Faber, S. M., Weiner, B. J., et al. 2007, ApJ, 660, L47

Noll, S., Burgarella, D., Giovannoli, E., et al. 2009, A\&A, 507, 1793

Oke, J. B. 1990, AJ, 99, 1621

Oliver, S., Frost, M., Farrah, D., et al. 2010, MNRAS, 405, 2279

Padmanabhan, N., Schlegel, D. J., Finkbeiner, D. P., et al. 2008, ApJ, 674, 1217

Pérez, F. \& Granger, B. E. 2007, Comput. Sci. Eng., 9, 21

Rieke, G. H., Young, E. T., Engelbracht, C. W., et al. 2004, ApJS, 154, 25

Roberts, M. S., \& Haynes, M. P. 1994, ARA\&A, 32, 115

Rohlfs, K., \& Wilson, T. L. 1996, Tools of Radio Astronomy, 127

Saintonge, A., Catinella, B., Cortese, L., et al. 2016, MNRAS, 462, 1749

Sault, R. J., Teuben, P. J., \& Wright, M. C. H. 1995, in Astronomical Data

Analysis Software and Systems IV, eds. R. A. Shaw, H. E. Payne, \&

J. J. E. Hayes, ASP Conf. Ser., 77, 433

Schiminovich, D., Wyder, T. K., Martin, D. C., et al. 2007, ApJS, 173, 315

Schlafly, E. F., \& Finkbeiner, D. P. 2011, ApJ, 737, 103

Schlegel, D. J., Finkbeiner, D. P., \& Davis, M. 1998, ApJ, 500, 525

Schreiber, C., Elbaz, D., Pannella, M., et al. 2016, A\&A, 589, A35

Schweizer, F. 1982, ApJ, 252, 455

Tody, D. 1993, in Astronomical Data Analysis Software and Systems II, eds

R. J. Hanisch, R. J. V. Brissenden, \& J. Barnes, ASP Conf. Ser., 52, 173

Toomre, A. 1977, in Evolution of Galaxies and Stellar Populations, eds.

B. M. Tinsley, R. B. G. Larson, \& D. Campbell, 401

Tran, H. D., Sirianni, M., Ford, H. C., et al. 2003, ApJ, 585, 750

van der Walt, S., Colbert, S. C., \& Varoquaux, G. 2011, Comput. Sci. Eng., 13, 22

York, D. G., Adelman, J., Anderson, Jr., J. E., et al. 2000, AJ, 120, 1579 